TRANSACTIONS OF THE

AMERICAN MATHEMATICAL SOCIETY

Volume 351, Number 8, Pages 3315-3349

S 0002-9947(99)02360-0

Article electronically published on April 8, 1999

\title{
NUMERATION SYSTEMS AND MARKOV PARTITIONS FROM SELF SIMILAR TILINGS
}

\author{
BRENDA PRAGGASTIS
}

\begin{abstract}
Using self similar tilings we represent the elements of $\mathbb{R}^{n}$ as digit expansions with digits in $\mathbb{R}^{n}$ being operated on by powers of an expansive linear map. We construct Markov partitions for hyperbolic toral automorphisms by considering a special class of self similar tilings modulo the integer lattice. We use the digit expansions inherited from these tilings to give a symbolic representation for the toral automorphisms.
\end{abstract}

Fractals and fractal tilings have captured the imaginations of a wide spectrum of disciplines. Computer generated images of fractal sets are displayed in public science centers, museums, and on the covers of scientific journals. Fractal tilings which have interesting properties are finding applications in many areas of mathematics. For example, number theorists have linked fractal tilings of $\mathbb{R}^{2}$ with numeration systems for $\mathbb{R}^{2}$ in complex bases [16], [8].

We will see that fractal self similar tilings of $\mathbb{R}^{n}$ provide natural building blocks for numeration systems of $\mathbb{R}^{n}$. These numeration systems generalize the 1-dimensional cases in [14],[10],[11] as well as the 2-dimensional cases mentioned above. Our motivation for studying fractal tilings comes from ergodic theory.

In [2] Adler and Weiss show that topological entropy is a complete invariant for metric equivalence of continuous ergodic automorphisms of the two-dimensional torus. Their method of proof is to construct a partition of the 2-torus which satisfies certain properties. The partition is called a Markov partition. By assigning each element of the partition a symbol, it is possible to assign each point in the 2-torus a bi-infinite sequence of symbols which corresponds to the orbit of the point. The objective is to represent the continuous dynamical system as a symbolic one in such a way that periodicity and transitivity is preserved in the representation.

In [4] Bowen shows that every Anosov diffeomorphism has a Markov partition. In particular every hyperbolic toral automorphism has a Markov partition. His construction uses a recursive definition which deforms rectangles in the stable and unstable directions. While existence is shown, the proof does not indicate an efficient way to actually construct the partitions. In [5] Bowen shows that in the case of the 3-torus the boundary sets of the Markov partition have fractional Hausdorff dimension. In [3] Bedford constructs examples of Markov partitions for the 3-torus and describes the sets as crinkly tin cans. In [6] Cawley generalizes Bowen's results to higher dimensional tori.

Since Markov partitions have properties which resemble those of self similar tilings and the boundary sets of these partitions have fractional dimension, it is

Received by the editors October 2, 1996.

1991 Mathematics Subject Classification. Primary 58F03, 34C35, 54H20.

(C)1999 American Mathematical Society 
natural to suggest that an explicit finite construction of Markov partitions for hyperbolic toral automorphisms may be given using fractal self similar tilings. Moreover, there is a natural way to represent points in a tiled space using a symbolic system. The representations correspond to digit expansions. The set of sequences of digits used in these expansions will in turn generate a symbolic dynamical system which is metrically similar to the continuous system defined by the corresponding toral automorphism.

\section{Subdividing AND SELF SIMILAR TILINGS}

In this first section we review the definition and properties of self similar tilings. Much of this information may be found in [17] and [10]. We then indicate how a self similar tiling of $\mathbb{R}^{n}$ provides a numeration system for $\mathbb{R}^{n}$. Let $X$ be a subset of $\mathbb{R}^{n}$ which is the closure of its interior.

Definition. A collection $\mathfrak{T}$ of compact subsets of $X$ is a tiling if it satisfies the following properties.

(1) The union of the sets in $\mathfrak{T}$ is equal to $X$.

(2) Each set in $\mathfrak{T}$ is the closure of its interior.

(3) Each compact set in $X$ intersects a finite number of sets in $\mathfrak{T}$. (In this case we say that $\mathfrak{T}$ is locally finite.)

(4) The interiors of the sets in $\mathfrak{T}$ are mutually disjoint.

The sets in $\mathfrak{T}$ are called tiles.

Suppose $G$ is a subgroup of $\mathbb{R}^{n}$. A tiling $\mathfrak{T}$ of $X$ is $\mathbf{G}$-finite if there exists a finite partition $\left\{\mathfrak{T}_{j}\right\}_{j \in J}$ of $\mathfrak{T}$ such that if $T \in \mathfrak{T}_{j}$, then

$$
\mathfrak{T}_{j} \subset\{T+g: g \in G\} .
$$

The collection $\left\{\mathfrak{T}_{j}\right\}_{j \in J}$ is a tile type partition for $\mathfrak{T}$.

Suppose $\mathfrak{T}$ is a $G$-finite tiling of $X$ with a tile type partition $\left\{\mathfrak{T}_{j}\right\}_{j \in J}$. If $T^{1}$, $T^{2} \in \mathfrak{T}_{j}$ for some $j \in J$, then we will say that $T^{1}$ and $T^{2}$ are of the same type. This implies that there exists $g \in G$ such that $T^{1}+g=T^{2}$. We say that $T^{2}$ is a G-translate of $T^{1}$. This does not mean that if $T^{1}, T^{2} \in \mathfrak{T}$ and $g \in G$ such that $T^{1}+g=T^{2}$, then $T^{1}$ and $T^{2}$ are of the same type. We will see that there will often exist tile type partitions which contain elements $\mathfrak{T}_{j_{1}}$ and $\mathfrak{T}_{j_{2}}$ such that every tile in $\mathfrak{T}_{j_{1}}$ is a $G$-translate of every tile in $\mathfrak{T}_{j_{2}}$. It follows that there are arbitrarily many different ways to define a tile type partition for a $G$-finite tiling. For now, let $\left\{\mathfrak{T}_{j}\right\}_{j \in J}$ define a tile type partition for $\mathfrak{T}$. Let $\mathfrak{P}$ be the set of all finite unions of tiles in $\mathfrak{T}$. Let $\mathfrak{B}$ be the set of all bounded subsets of $X$. Note that $\mathfrak{P} \subset \mathfrak{B}$.

Definition. Let $P^{1} ; P^{2} \in \mathfrak{P}$, then there is a finite collection of tiles $\left\{T^{k}\right\}_{k \in K}$ such that $P^{1}=\bigcup_{k \in K} T^{k}$. The sets $P^{1}$ and $P^{2}$ have the same pattern if there exists $g \in G$ such that $P^{1}+g=\bigcup_{k \in K} T^{k}+g=P^{2}$ and for each $k \in K, T^{k}+g$ is a tile in $\mathfrak{T}$ of the same type as $T^{k}$. In particular tiles of the same type have the same pattern. If $U \in \mathfrak{B}$, then there is a finite union of tiles $P \in \mathfrak{P}$ such that $U \subset P$. If $g \in G$, then $U$ and $U+g$ have the same pattern if for some $P \in \mathfrak{P}$ such that $U \subset P$ we have that $P+g \in \mathfrak{P}$ and $P+g$ and $P$ have the same pattern. If $V$ is a subset of $X$ and $U \in \mathfrak{B}$, then $V$ contains the pattern of $U$ if for some $g \in G$, $U+g \subset V$ and $U+g$ has the same pattern as $U$.

Let $\phi$ be an expansive linear map on $\mathbb{R}^{n}$ such that $\phi X=X$. To be expansive means that the eigenvalues for $\phi$ all have modulus greater than 1 . We adapt a 
norm for $\mathbb{R}^{n}$ which reflects the expansiveness of $\phi$ as in [12]. More specifically, let $\left\{\lambda_{i}\right\}_{i=1}^{l}$ be the eigenvalues of $\phi$ ordered so that

$$
1<\left|\lambda_{1}\right| \leq\left|\lambda_{2}\right| \leq \cdots \leq\left|\lambda_{l}\right|
$$

Choose $\varrho$ such that $1<\varrho<\left|\lambda_{1}\right|$. We choose a norm $\|\cdot\|$ so that for all $x \in \mathbb{R}^{n}$

$$
\begin{gathered}
\|x\|<\varrho\|x\| \leq\|\phi x\| \text { and } \\
\left\|\phi^{-1} x\right\| \leq \varrho^{-1}\|x\|<\|x\| .
\end{gathered}
$$

Definition. A $G$-finite tiling $\mathfrak{T}$ with tile type partition $\left\{\mathfrak{T}_{j}\right\}_{j \in J}$ has a finite number of local patterns if, for each $\delta>0$ we have that the set

$$
\{P \in \mathfrak{P}: \operatorname{diameter}(P)<\delta\}
$$

contains a finite number of distinct patterns.

A slightly stronger restriction on $\mathfrak{T}$ is that $\mathfrak{T}$ has only a finite number of local patterns and these patterns are scattered uniformly throughout the tiling. Let $B_{r}(x)$ denote the open ball of radius $r$ about $x$.

Definition. A $G$-finite tiling $\mathfrak{T}$ with tile type partition $\left\{\mathfrak{T}_{j}\right\}_{j \in J}$ is called quasiperiodic if for each $r>0$ there exists $R=R(r)>0$ such that every open ball of radius $R$ contained in $X$ contains the pattern of $B_{r}(x) \cap X$ for all $x \in X$.

Let $G$ be a subgroup of $\mathbb{R}^{n}$ such that $\phi G=G$.

Definition. A $G$-finite tiling $\mathfrak{T}$ of $X$ with tile type partition $\left\{\mathfrak{T}_{j}\right\}_{j \in J}$ is subdividing with expansion map $\phi$, if for each $T \in \mathfrak{T}, \phi T$ is a finite union of tiles and if $T$ and $T^{\prime}$ are tiles of the same type, then $\phi T$ and $\phi T^{\prime}$ have the same pattern. For each $T \in \mathfrak{T}_{j}$ we may think of the pattern of $\phi T$ as defining a subdivision rule for $\mathfrak{T}_{j}$. A quasi-periodic subdividing tiling of $\mathfrak{T}$ of $X$ with expansion map $\phi$ is called a self similar tiling.

Example 1. Let $\phi$ be the expansive map on $\mathbb{R}$ given by multiplication by

$$
\lambda=\frac{1+\sqrt{5}}{2} \text {. }
$$

Note that $\lambda$ is a zero of $x^{2}-x-1$. Let $X^{+}=[0, \infty)$. We construct a $\mathbb{Z}[\lambda]$ finite subdividing tiling $\mathfrak{T}$ of $X^{+}$with expansion map $\phi$. Let $T_{\mathbf{A}}=[0,1]$ and $T_{\mathbf{B}}=[0, \lambda-1]$. We will partition $\mathfrak{T}$ into two sets $\mathfrak{T}_{\mathbf{A}}$ and $\mathfrak{T}_{\mathbf{B}}$. The tiles in $\mathfrak{T}_{\mathbf{A}}$ will be $\mathbb{Z}[\lambda]$-translates of $T_{\mathbf{A}}$ and the tiles in $\mathfrak{T}_{\mathbf{B}}$ will be $\mathbb{Z}[\lambda]$-translates of $T_{\mathbf{B}}$. We define $\mathfrak{T}$ by inductively defining $\mathfrak{T}_{\mathbf{A}}$ and $\mathfrak{T}_{\mathbf{B}}$. Let $T_{\mathbf{A}} \in \mathfrak{T}_{\mathbf{A}}$. If $x \in \mathbb{Z}[\lambda]$ and $T_{\mathbf{A}}+x \in \mathfrak{T}_{\mathbf{A}}$, then

$$
\begin{aligned}
\phi\left(T_{\mathbf{A}}+x\right) & =[0, \lambda]+\lambda x \\
& =([0,1]+\lambda x) \cup([1, \lambda]+\lambda x) \\
& =\left(T_{\mathbf{A}}+\lambda x\right) \cup\left(T_{\mathbf{B}}+1+\lambda x\right) .
\end{aligned}
$$

Let $T_{\mathbf{A}}+\lambda x \in \mathfrak{T}_{\mathbf{A}}$ and let $T_{\mathbf{B}}+1+\lambda x \in \mathfrak{T}_{\mathbf{B}}$. If $y \in \mathbb{Z}[\lambda]$ and $T_{\mathbf{B}}+y \in \mathfrak{T}_{\mathbf{B}}$, then

$$
\begin{aligned}
\phi\left(T_{\mathbf{B}}+y\right) & =\left[0, \lambda^{2}-\lambda\right]+\lambda y \\
& =[0,1]+\lambda y \\
& =T_{\mathbf{A}}+\lambda y .
\end{aligned}
$$


Let $T_{\mathbf{A}}+\lambda y \in \mathfrak{T}_{\mathbf{A}}$. The tiling $\mathfrak{T}$ is a $\mathbb{Z}[\lambda]$-finite subdividing tiling of $X^{+}$with expansion map $\phi$ and tile type partition $\left\{\mathfrak{T}_{\mathbf{A}}, \mathfrak{T}_{\mathbf{B}}\right\}$.

We can represent the subdivision rules for $\mathfrak{T}$ in terms of a substitution map. Let $\mathbf{A}$ represent a tile in $\mathfrak{T}_{\mathbf{A}}$ and $\mathbf{B}$ represent a tile in $\mathfrak{T}_{\mathbf{B}}$. Define a substitution map $\theta$ on the words in $\{\mathbf{A}, \mathbf{B}\}$ by letting $\theta(\mathbf{A})=\mathbf{A B}$ and letting $\theta(\mathbf{B})=\mathbf{A}$. We define $\theta$ on each finite or infinite string of symbols in $\{\mathbf{A}, \mathbf{B}\}$ by applying it to each symbol in the string. We see that $\mathfrak{T}$ is represented by the fixed word

$$
w=\mathbf{A B A} \mathbf{A B A B A} \mathbf{A B A A B} \ldots
$$

That is, $\theta w=w$.

Example 1 illustrates a subdividing tiling which is actually self similar. We will prove this later. For now the main thing to note is that $T_{\mathbf{A}} \subset \phi T_{\mathbf{A}}$.

We now assume that $\mathfrak{T}$ is a subdividing tiling of $X$ with expansive map $\phi$. Let $\left\{\mathfrak{T}_{j}\right\}_{j \in J}$ be a tile type partition for $\mathfrak{T}$. For each tile $T$ let $\partial T$ denote the boundary of $T$. Let $\partial \mathfrak{T}=\bigcup_{T \in T} \partial T$ be the boundary of $\mathfrak{T}$. Let $\mu$ be Lebesgue measure on $\mathbb{R}^{n}$.

Proposition 1.1. The Lebesgue measure of the boundary of $\mathfrak{T}$ is 0 .

Proof. Suppose $\mu(\partial \mathfrak{T}) \neq 0$. Then since $\mathfrak{T}$ is locally finite for some tile $T$, we have $\mu(\partial T) \neq 0$. Since $\mathfrak{T}$ contains only a finite number of tile types, there exists

$$
\delta=\max \left\{\frac{\mu(\partial T)}{\mu(T)}: T \in \mathfrak{T}\right\}<1
$$

It follows that there exists a tile $T_{0}$ such that $\mu\left(\partial T_{0}\right)=\delta \mu\left(T_{0}\right)$, and for all $T^{\prime} \in \mathfrak{T}$ we have $\mu\left(\partial T^{\prime}\right) \leq \delta \mu\left(T^{\prime}\right)$.

Note that if $T^{1}$ and $T^{2}$ are two distinct tiles, then

$$
\begin{aligned}
\mu\left(\partial\left(T^{1} \cup T^{2}\right)\right) & \leq \mu\left(\partial T^{1}\right)+\mu\left(\partial T^{2}\right)-\mu\left(\partial T^{1} \cap \partial T^{2}\right) \\
& \leq \delta \mu\left(T^{1}\right)+\delta \mu\left(T^{2}\right)-\mu\left(\partial T^{1} \cap \partial T^{2}\right) \\
& =\delta\left(\mu\left(T^{1} \cup T^{2}\right)+\mu\left(T^{1} \cap T^{2}\right)\right)-\mu\left(\partial T^{1} \cap \partial T^{2}\right) \\
& =\delta \mu\left(T^{1} \cup T^{2}\right)+(\delta-1) \mu\left(\partial T^{1} \cap \partial T^{2}\right) \\
& \leq \delta \mu\left(T^{1} \cup T^{2}\right)
\end{aligned}
$$

since $T^{1} \cap T^{2}=\partial T^{1} \cap \partial T^{2}$. Applying induction we find for any finite collection of distinct tiles $\left\{T^{k}\right\}_{k=1}^{L}$ that

$$
\mu\left(\partial \bigcup_{k=1}^{L} T^{k}\right) \leq \delta \mu\left(\bigcup_{k=1}^{L} T^{k}\right) .
$$

Since $\phi$ is expansive we choose $N_{0}$ such that for all $N \geq N_{0}$ we have $\operatorname{Int}\left(\phi^{N} T_{0}\right)$ contains a tile. Let $P_{N}$ be the element of $\mathfrak{P}$ consisting of just those tiles in $\phi^{N} T_{0}$ which intersect the set $\phi^{N}\left(\partial T_{0}\right)$. Since $\phi$ is a homeomorphism, $\phi^{N}\left(\partial T_{0}\right)$ is just the set $\partial\left(\phi^{N} T_{0}\right)$. We have $\mu\left(P_{N}\right)<\mu\left(\phi^{N} T_{0}\right)$ for all $N \geq N_{0}$ since $\phi^{N} T_{0}$ contains a tile which does not intersect its boundary. By the previous paragraph we have 


$$
\begin{aligned}
\mu\left(\partial \phi^{N} T_{0}\right) \leq \delta \mu\left(P_{N}\right) \text { since } \partial \phi^{N} T_{0} & \subset \partial P_{N} \text {. So } \\
\delta & =\frac{\left|\operatorname{det} \phi^{N}\right| \cdot \mu\left(\partial T_{0}\right)}{\left|\operatorname{det} \phi^{N}\right| \cdot \mu\left(T_{0}\right)} \\
& =\frac{\mu\left(\phi^{N} \partial T_{0}\right)}{\mu\left(\phi^{N} T_{0}\right)} \\
& \leq \frac{\delta \mu\left(P_{N}\right)}{\mu\left(\phi^{N} T_{0}\right)} \\
& <\delta .
\end{aligned}
$$

The contradiction implies that $\mu(\partial T)=0$ for each tile $T$.

Definition. A $G$-finite subdividing tiling $\mathfrak{T}$ with expansive map $\phi$ and tile type partition $\left\{\mathfrak{T}_{j}\right\}_{j \in J}$ is mixing if for each $T \in \mathfrak{T}$ and $P \in \mathfrak{P}$ there exists $N_{0} \geq 0$ such that for all $N \geq N_{0}$ we have that $\phi^{N}(T)$ contains the pattern of $P$.

The following fact was also noted by Kenyon in [9]. The proof may be found in [15].

Proposition 1.2. Let $\mathfrak{T}$ be a $G$-finite subdividing tiling of $X$ with expansive map $\phi$ and tile type partition $\left\{\mathfrak{T}_{j}\right\}_{j \in J}$. Then $\mathfrak{T}$ is quasi-periodic if and only if $\mathfrak{T}$ is mixing and has a finite number of local patterns.

Example 2. Let $\mathfrak{T}$ be the subdividing tiling of $X^{+}$in Example 1. Let $\delta>0$. There are only a finite number of ways to arrange almost disjoint $\mathbb{Z}[\lambda]$-translates of $T_{\mathbf{A}}$ and $T_{\mathbf{B}}$ in $X^{+}$so that their union is connected and has diameter less than $\delta$. So $\mathfrak{T}$ has a finite number of local patterns. Let $P$ be a finite union of tiles in $\mathfrak{T}$. For some $N \geq 0$ we have $P \subset \phi^{N} T_{\mathbf{A}}$. Hence for all $T \in \mathfrak{T}_{\mathbf{A}}$ we have that $\phi^{N} T$ contains the pattern of $P$. If $T^{1} \in \mathfrak{T}$, then $\phi T^{1}$ contains a tile in $\mathfrak{T}_{\mathbf{A}}$ so $\phi^{N+1} T^{1}$ contains the pattern of $P$. It follows from Proposition 1.2 that $\mathfrak{T}$ is self similar.

Since $\mathfrak{T}$ has a finite number of tile types, we may record the subdivision rules for $\mathfrak{T}$ in a finite manner. We will use a graph to indicate the subdivision rules for $\mathfrak{T}$. The generic definitions for graphs and the objects related to graphs given below are adapted from [13].

A graph $\Gamma$ consists of a finite set of vertices $\mathcal{V}$ together with a finite set of edges $\mathcal{E}$. The edge $\alpha$ has a source $s(\alpha)$ and a target $t(\alpha)$. If $I$ is a set of consecutive integers in $\mathbb{Z}$, then a sequence (or finite sequence or bi-infinite sequence) of edges $\left\{\eta^{k}\right\}_{k \in I}$ is called a path in $\Gamma$ if for each $k, k+1 \in I$ we have $t\left(\eta^{k}\right)=s\left(\eta^{k+1}\right)$. If $\left\{\eta^{k}\right\}_{k=-N}^{\infty}$ is a path in $\Gamma$, then $s\left(\eta^{-N}\right)$ is the source of the path. If $\left\{\eta^{k}\right\}_{k=-\infty}^{M}$ is a path in $\Gamma$, then $t\left(\eta^{M}\right)$ is the target of the path. A path $\left\{\eta^{k}\right\}_{k=-N}^{M}$ has both a source $s\left(\eta^{-N}\right)$ and a target $t\left(\eta^{M}\right)$. A path $\left\{\eta^{k}\right\}_{k \in \mathbb{Z}}$ has neither a source nor a target. If $I$ is a finite set of consecutive integers, then the length of a path $\left\{\eta^{k}\right\}_{k \in I}$ is $|I|$.

If $\eta$ and $\epsilon$ are two paths in $\Gamma$, then we shall say that $\eta$ equals $\epsilon$ if $\eta=\left\{\eta^{k}\right\}_{k \in I}$ and $\epsilon=\left\{\epsilon^{k}\right\}_{k \in I}$ for some consecutive sequence of integers $I$ and for each $k \in I$ we have $\eta^{k}=\epsilon^{k}$. That is, the two paths must be indexed by the same set and each pair of edges $\eta^{k}$ and $\epsilon^{k}$ with the same index are actually the same edge. If $\eta$ is not equal to $\epsilon$, then we shall say that the paths are distinct.

Definition. The subdivision graph $\Gamma$ for $\mathfrak{T}$ has a finite set of vertices $\mathcal{V}$ indexed by $J$ and edges $\mathcal{E}$. If $v_{j_{1}}$ and $v_{j_{2}}$ are vertices in $\mathcal{V}$, then there are exactly $M_{j_{1} j_{2}}$ 
edges with source $v_{j_{1}}$ and target $v_{j_{2}}$ if and only if for each $T \in \mathfrak{T}_{j_{1}}$ there are exactly $M_{j_{1} j_{2}}$ distinct elements of $\mathfrak{T}_{j_{2}}$ contained in $\phi T$. Since $\mathfrak{T}$ is subdividing, the number $M_{j_{1} j_{2}}$ is well-defined for each pair $j_{1}$ and $j_{2}$. The transition matrix $M$ is given by $\left\{M_{j_{1}, j_{2}}\right\}_{\left(j_{1}, j_{2}\right) \in J \times J}$. Note that if $\mathfrak{T}$ is mixing, then $M$ is aperiodic.

Let $\Gamma$ be the subdivision graph for $\mathfrak{T}$. For each $j_{1}, j_{2} \in J$ let $\mathcal{E}_{j_{1}}^{j_{2}}$ be the subset of edges in $\mathcal{E}$ which have source $v_{j_{1}}$ and target $v_{j_{2}}$. Let $\mathcal{E}_{j_{1}}$ be the subset of edges in $\mathcal{E}$ which have source $v_{j_{1}}$ and let $\mathcal{E}^{j_{2}}$ be the subset of edges in $\mathcal{E}$ which have target $v_{j_{2}}$. We use the edges in $\mathcal{E}_{j_{1}}$ to index the tiles in $\phi T$ for each $T \in \mathfrak{T}_{j_{1}}$. That is, we write

$$
\phi T=\bigcup_{\alpha \in \mathcal{E}_{j_{1}}} T^{\alpha}
$$

for the unique finite union of tiles found in $\phi T$ such that if $t(\alpha)=v_{k_{\alpha}}$, then $T^{\alpha} \in \mathfrak{T}_{k_{\alpha}}$.

Let $\left\{T_{j}\right\}_{j \in J}$ be a representative set of tiles for $\left\{\mathfrak{T}_{j}\right\}_{j \in J}$ such that $T_{j} \in \mathfrak{T}_{j}$. Fix a point $x_{j} \in T_{j}$. For each $T_{j}+g \in \mathfrak{T}_{j}$ define

$$
d\left(T_{j}+g\right)=x_{j}+g .
$$

The point $d\left(T_{j}+g\right)$ is called a positional point for $T_{j}+g$ and $d(\mathfrak{T})$ is a set of positional points for $\mathfrak{T}$. Suppose $T^{0} \in \mathfrak{T}_{j_{0}}$ and $T^{1} \in \mathfrak{T}_{j_{1}}$ such that $T^{1} \subset \phi T^{0}$. We record the relative position of $T^{1}$ in $\phi T^{0}$ by noting the difference

$$
d\left(T^{1}\right)-\phi d\left(T^{0}\right) .
$$

Let $T^{2} \in \mathfrak{T}_{j_{0}}$. Then there exists a unique $T^{3} \in \mathfrak{T}_{j_{1}}$ such that $T^{3} \subset \phi T^{2}$ and

$$
d\left(T^{3}\right)-\phi d\left(T^{2}\right)=d\left(T^{1}\right)-\phi d\left(T^{0}\right) .
$$

We have in this case

$$
d\left(T^{3}\right)=\phi d\left(T^{2}\right)+\left(d\left(T^{1}\right)-\phi d\left(T^{0}\right)\right) .
$$

This is one way of saying that $T^{3}$ is in the same relative position in $\phi T^{2}$ as $T^{1}$ is in $\phi T^{0}$.

Definition. The label map $L_{d}$ assigns to each edge $\alpha \in \mathcal{E}$ a vector in $\mathbb{R}^{n}$. If $T \in \mathfrak{T}_{j}$ and $\left\{T^{\alpha}\right\}_{\alpha \in \mathcal{E}_{j}}$ is the unique set of tiles in $\mathfrak{T}$ such that

$$
\phi T=\bigcup_{\alpha \in \mathcal{E}_{j}} T^{\alpha},
$$

then $L_{d}(\alpha)=d\left(T^{\alpha}\right)-\phi d(T)$ for each $\alpha \in \mathcal{E}_{j}$.

The pair $\left(\Gamma, L_{d}\right)$ completely describes the subdivision rules for $\mathfrak{T}$. If $T \in \mathfrak{T}_{j}$, then for each $\alpha \in \mathcal{E}_{j}^{k}$ there is a unique tile $T^{\prime} \in \mathfrak{T}_{k}$ such that $T^{\prime} \subset \phi T$ and

$$
d\left(T^{\prime}\right)=\phi d(T)+L_{d}(\alpha) .
$$

Example 3. Let $\mathfrak{T}$ be the subdividing tiling of $X^{+}=[0, \infty)$ with expansive map $\phi$ given in Example 1. For each $T \in \mathfrak{T}$ let $d(T)$ be the left endpoint of $T$. The subdivision graph for $\mathfrak{T}$ is in Figure 1. Each edge $\alpha$ is labeled with $L_{d}(\alpha)$.

Definition. Let $\Theta_{d}: \mathcal{E} \rightarrow \mathcal{V} \times \mathcal{V} \times \mathbb{R}^{n}$ such that for each $\alpha \in \mathcal{E}$ we have

$$
\Theta_{d}(\alpha)=\left(s(\alpha), t(\alpha), L_{d}(\alpha)\right) \text {. }
$$




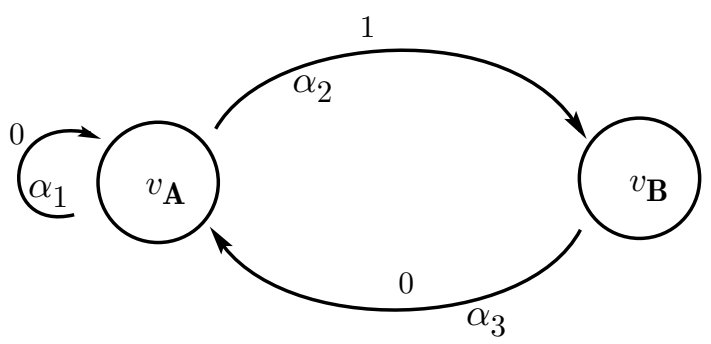

Figure 1. The subdivision graph for Example 1

The map $\Theta_{d}$ is a one-one assignment. For suppose $\alpha, \alpha^{\prime}$ are edges in $\mathcal{E}_{j}^{k}$ such that $\Theta_{d}(\alpha)=\Theta_{d}\left(\alpha^{\prime}\right)$. Then for each $T \in \mathfrak{T}_{j}$ there exists a unique $T^{\alpha} \in \mathfrak{T}_{k}$ such that

$$
d\left(T^{\alpha}\right)=\phi d(T)+L_{d}(\alpha) .
$$

Similarly, there is a unique $T^{\alpha^{\prime}} \in \mathfrak{T}_{k}$ such that

$$
d\left(T^{\alpha^{\prime}}\right)=\phi d(T)+L_{d}\left(\alpha^{\prime}\right) .
$$

But $L_{d}(\alpha)=L_{d}\left(\alpha^{\prime}\right)$ and $T^{\alpha}, T^{\alpha^{\prime}} \in \mathfrak{T}_{k}$. So $T^{\alpha}=T^{\alpha^{\prime}}$. But edges in $\mathcal{E}_{j}$ refer to distinct $\mathfrak{T}$ tiles in $\phi T$ so $\alpha=\alpha^{\prime}$. We find for each triple $\left(v_{j}, v_{k}, x\right) \in \Theta_{d}(\mathcal{E})$ there is a unique $\alpha \in \mathcal{E}$ such that $\Theta_{d}(\alpha)=\left(v_{j}, v_{k}, x\right)$. In this case we write $\Theta_{d}^{-1}\left(v_{j}, v_{k}, x\right)=\alpha$.

In [17] and [10] the tiles in a self similar tiling are assigned control points. The control points reflect the similarity properties of the tiling. By considering all $\phi$ preimages of control points one may recover the tiling. By studying the behavior of control points under the map $\phi$, Thurston and Kenyon are able to analyze the expansive maps associated to self similar tilings in terms of their eigenvalues.

There are many ways to define control points. For the purpose of generality we will want our definition to apply to any subdividing tiling. We begin by defining a tile map for $\mathfrak{T}$.

Definition. A map $\gamma: \mathfrak{T} \rightarrow \mathfrak{T}$ is a tile map for $\mathfrak{T}$ if for each $T \in \mathfrak{T}_{j}, j \in J$ and for each $g \in G$ such that $T+g \in \mathfrak{T}_{j}$ we have $\gamma(T) \subset \phi T$ and $\gamma(T+g)=\gamma(T)+\phi g$.

We construct a tile map for $\mathfrak{T}$ by fixing one tile $T_{j} \in \mathfrak{T}_{j}$ for each $j \in J$ and defining $\gamma\left(T_{j}\right)$ to be some tile in $\mathfrak{T}$ contained in $\phi\left(T_{j}\right)$. For each $T_{j}+g \in \mathfrak{T}_{j}$ let $\gamma\left(T_{j}+g\right)=\gamma\left(T_{j}\right)+\phi g$. Note that for each $k \geq 0$

$$
\phi^{-k} \gamma^{k}(T) \subset T \text {. }
$$

Since $\phi$ is expansive,

$$
\bigcap_{k=0}^{\infty} \phi^{-k} \gamma^{k}(T)
$$

is a decreasing intersection of compact sets with diameters tending to 0 . Hence there exists a unique point $c(T)$ contained in this intersection. We call $c(T)$ a control point for $T$ and $c(\mathfrak{T})$ the set of control points for $\mathfrak{T}$ induced by $\gamma$. 
Proposition 1.3. Let $c: \mathfrak{T} \rightarrow X$ be the map which assigns to each $T \in \mathfrak{T}$ the unique point

$$
\{c(T)\}=\bigcap_{k=0}^{\infty} \phi^{-k} \gamma^{k}(T) .
$$

(1) If $T \in \mathfrak{T}$, then $\phi c(T)=c(\gamma(T))$.

(2) If $T^{1} \in \mathfrak{T}_{j}$ and $T^{2} \in \mathfrak{T}_{j}$, then

$$
T^{1}+c\left(T^{2}\right)-c\left(T^{1}\right)=T^{2} .
$$

Corollary 1.4. If $T^{1}, T^{2} \in \mathfrak{T}_{j}$, then $c\left(T^{1}\right)=c\left(T^{2}\right)$ if and only if $T^{1}=T^{2}$.

Note that if $T^{1}, T^{2} \in \mathfrak{T}_{j}$ and $T^{1}+g=T^{2}$, then $c\left(T^{1}\right)+g=c\left(T^{2}\right)$. So $c(\mathfrak{T})$ is a set of positional points for $\mathfrak{T}$. Since $c$ depends only on $\gamma$, we call $L=L_{c}$ the label map induced by $\gamma$. Similarly, we let $\Theta=\Theta_{c}$ be the one-one assignment

$$
\Theta(\alpha)=(s(\alpha), t(\alpha), L(\alpha))
$$

for each edge $\alpha \in \mathcal{E}$. The vectors which label the edges in $\Gamma$ according to the label map $L$ provide a set of digits for a numeration system of $X$. The balance of this section demonstrates how this is done.

Let $x \in T^{0} \in \mathfrak{T}_{j_{0}}, j_{0} \in J$. For each $k \geq 1$ inductively define $T^{k}$ to be some tile such that $\phi^{k} x \in T^{k} \subset \phi T^{k-1}$. Then $\left\{\phi^{-k} T^{k}\right\}_{k=0}^{\infty}$ is a decreasing sequence of compact subsets with diameters tending to 0 and $x$ is the unique point in $\bigcap_{k=0}^{\infty} \phi^{-k} T^{k}$. We say that $\left\{T^{k}\right\}_{k=0}^{\infty}$ converges to the point $\mathbf{x}$.

Theorem 1.5. Let $\mathfrak{T}$ be a subdividing tiling of $X$ with expansion map $\phi$ and tile type partition $\left\{\mathfrak{T}_{j}\right\}_{j \in J}$. Let $\Gamma$ be the subdivision graph for $\mathfrak{T}$ and $c(\mathfrak{T})$ a set of control points for $\mathfrak{T}$. For $\mu$-almost every $x \in X$ there is a unique sequence of tiles converging to $x$. If $\mathfrak{T}$ has a finite number of local patterns, then there is a uniform bound on the number of sequences of tiles converging to $x$.

Suppose that $\left\{T^{k}\right\}_{k=0}^{\infty}$ is a sequence of tiles converging to $x$. Let $j_{k}$ be the element of $J$ such that $T^{k} \in \mathfrak{T}_{j_{k}}$ for each $k \geq 0$. We have

$$
x=c\left(T^{0}\right)+\sum_{k=1}^{\infty} \phi^{-k}\left(c\left(T^{k}\right)-\phi c\left(T^{k-1}\right)\right) .
$$

Moreover there exists a unique path $\left\{\eta^{k}\right\}_{k=1}^{\infty}$ in $\Gamma$ defined by

$$
\eta^{k}=\Theta^{-1}\left(v_{j_{k-1}}, v_{j_{k}}, c\left(T^{k}\right)-\phi c\left(T^{k-1}\right)\right)
$$

and

$$
x=c\left(T^{0}\right)+\sum_{k=1}^{\infty} \phi^{-k} L\left(\eta^{k}\right) .
$$

Conversely, suppose $\eta=\left\{\eta^{k}\right\}_{k=1}^{\infty}$ is a path in $\Gamma$ with source $v_{j_{0}}$ and $x \in T^{0} \in \mathfrak{T}_{j_{0}}$ such that

$$
x=c\left(T^{0}\right)+\sum_{k=1}^{\infty} \phi^{-k} L\left(\eta^{k}\right)
$$

and for each $k \geq 1$

$$
\Theta\left(\eta^{k}\right)=\left(v_{j_{k-1}}, v_{j_{k}}, L\left(\eta^{k}\right)\right)
$$


Then there is a unique sequence of tiles $\left\{T^{k}\right\}_{k=0}^{\infty}$ converging to $x$ such that $T^{k} \in \mathfrak{T}_{j_{k}}$ and

$$
c\left(T^{k}\right)=\phi c\left(T^{k-1}\right)+L\left(\eta^{k}\right)
$$

for each $k \geq 1$.

Proof. Let $x \in X$. By the above discussion we may inductively define some sequence of tiles $\left\{T^{k}\right\}_{k=0}^{\infty}$ which converges to $x$. Suppose that $\left\{T_{1}^{k}\right\}_{k=0}^{\infty}$ and $\left\{T_{2}^{k}\right\}_{k=0}^{\infty}$ are two distinct sequences of tiles which converge to $x$. For some minimal $N \geq 1$ we have $\phi^{N} x \in T_{1}^{N} \cap T_{2}^{N}$ and $T_{1}^{N} \neq T_{2}^{N}$. Since $T_{i}^{N+k} \subset \phi^{k} T_{i}^{N}, i \in\{1,2\}$, we have $T_{1}^{N+k} \neq T_{2}^{N+k}$ for all $k \geq 0$. Hence $\phi^{N+k} x \in \partial \mathfrak{T}$ for all $k \geq 0$. By Proposition 1.1, $\mu(\partial \mathfrak{T})=0$. Since $\phi$ is a linear map, $\mu\left(\bigcup_{k=0}^{\infty} \phi^{-k} \partial \mathfrak{T}\right)=0$. Hence for $\mu$-almost every $x \in X$ there is a unique sequence of tiles converging to $x$. In any case the number of distinct sequences of tiles converging to $x$ is bounded by the number of tiles in $\mathfrak{T}$ which may share a point in common. If $\mathfrak{T}$ has a finite number of local patterns, then there exists a uniform bound on this number.

Suppose $\left\{T^{k}\right\}_{k=0}^{\infty}$ converges to $x$ and $T^{k} \in \mathfrak{T}_{j_{k}}, j_{k} \in J$. Let $D$ be the maximum diameter of any tile. Since $x \in T^{0}$, we have

$$
\left\|x-c\left(T^{0}\right)\right\| \leq D .
$$

Similarly for each $N \geq 1$, we have $\phi^{N} x \in T^{N}$ so

$$
\left\|\phi^{N} x-\phi^{N} c\left(T^{0}\right)-\sum_{k=1}^{N} \phi^{N-k}\left(c\left(T^{k}\right)-\phi c\left(T^{k-1}\right)\right)\right\|=\left\|\phi^{N} x-c\left(T^{N}\right)\right\| \leq D .
$$

Recall that, for each $y \in \mathbb{R}^{n}$, we have $\left\|\phi^{-N} y\right\| \leq \varrho^{-N}\|y\|$, so

$$
\left\|x-c\left(T^{0}\right)-\sum_{k=1}^{\infty} \phi^{-k}\left(c\left(T^{k}\right)-\phi c\left(T^{k-1}\right)\right)\right\| \leq \varrho^{-N} D .
$$

Letting $N$ tend to infinity we obtain Equation (1).

For each $k \geq 1$ we have $T^{k} \subset \phi T^{k-1}$ so there exists an edge $\eta^{k} \in \mathcal{E}_{j_{k-1}}^{j_{k}}$ such that $\eta^{k}=\Theta^{-1}\left(v_{j_{k-1}}, v_{j_{k}}, c\left(T^{k}\right)-\phi c\left(T^{k-1}\right)\right)$. Since $t\left(\eta^{k}\right)=s\left(\eta^{k+1}\right)$ for each $k \geq 1$, the sequence $\left\{\eta^{k}\right\}_{k=1}^{\infty}$ is a path in $\Gamma$ with source $s\left(\eta^{1}\right)=v_{j_{0}}$. The map $\Theta^{-1}$ is well defined so $\left\{\eta^{k}\right\}_{k=1}^{\infty}$ is uniquely defined by $\left\{T^{k}\right\}_{k=1}^{\infty}$. Finally, we replace $c\left(T^{k}\right)-\phi c\left(T^{k-1}\right)$ with $L\left(\eta^{k}\right)$ in (1) to obtain (2).

Suppose now that $\eta=\left\{\eta^{k}\right\}_{k=1}^{\infty}$ is a path in $\Gamma$ with source $v_{j_{0}}$ and $x \in T^{0}$ such that

$$
x=c\left(T^{0}\right)+\sum_{k=1}^{\infty} \phi^{-k} L\left(\eta^{k}\right)
$$

and

$$
\Theta\left(\eta^{k}\right)=\left(v_{j_{k-1}}, v_{j_{k}}, L\left(\eta^{k}\right)\right)
$$

We apply induction on $k$ to construct $\left\{T^{k}\right\}_{k=1}^{\infty}$. Since $\Theta\left(\eta^{1}\right)=\left(v_{j_{0}}, v_{j_{1}}, L\left(\eta^{1}\right)\right)$, there exists a unique tile $T^{1} \subset \phi T^{0}$ such that $T^{1} \in \mathfrak{T}_{j_{1}}$ and

$$
c\left(T^{1}\right)=\phi c\left(T^{0}\right)+L\left(\eta^{1}\right) .
$$

Suppose that $T^{N} \subset \phi T^{N-1}, T^{N} \in \mathfrak{T}_{j_{N}}$ and

$$
c\left(T^{N}\right)=\phi c\left(T^{N-1}\right)+L\left(\eta^{N}\right) .
$$


Since $\Theta\left(\eta^{N+1}\right)=\left(v_{j_{N}}, v_{j_{N+1}}, L\left(\eta^{N+1}\right)\right)$ there is a unique tile $T^{N+1} \subset \phi T^{N}$ such that $T^{N+1} \in \mathfrak{T}_{j_{N+1}}$ and

$$
c\left(T^{N+1}\right)=\phi c\left(T^{N}\right)+L\left(\eta^{N+1}\right) .
$$

In this way we construct a unique sequence of tiles $\left\{T^{k}\right\}_{k=1}^{\infty}$. Since

$$
\bigcap_{k=0}^{\infty} \phi^{-k} T^{k}
$$

is a decreasing intersection of compact sets with diameters tending to 0 , there exists a unique point $y \in \phi^{-k} T^{k}$ for all $k \geq 0$ such that $\left\{T^{k}\right\}_{k=0}^{\infty}$ converges to $y$. By the first part of the argument

$$
\begin{aligned}
y & =c\left(T^{0}\right)+\sum_{k=1}^{\infty} \phi^{-k}\left(c\left(T^{k}\right)-\phi c\left(T^{k-1}\right)\right) \\
& =c\left(T^{0}\right)+\sum_{k=1}^{\infty} \phi^{-k} L\left(\eta^{k}\right)=x
\end{aligned}
$$

Corollary 1.6. Let $j \in J$ and $T \in \mathfrak{T}_{j}$. Then

$$
T-c(T)=\left\{\sum_{k=1}^{\infty} \phi^{-k} L\left(\eta^{k}\right):\left\{\eta^{k}\right\}_{k=1}^{\infty} \text { is a path in } \Gamma \text { with source } v_{j}\right\} .
$$

Corollary 1.7. If $\mathfrak{T}$ has a finite number of local patterns, then there exists a uniform bound on the number of paths $\left\{\eta^{k}\right\}_{k=1}^{\infty}$ in $\Gamma$ for which $x=\sum_{k=1}^{\infty} \phi^{-k} L\left(\eta^{k}\right)$, for all $x \in X$.

Corollary 1.8. Suppose $\eta=\left\{\eta^{k}\right\}_{k=1}^{M}$ and $\epsilon=\left\{\epsilon^{k}\right\}_{k=1}^{M}$ are two paths in $\Gamma$ with the same source $v_{j_{0}}$ and target $v_{j_{1}}$. Then

$$
\sum_{k=1}^{M} \phi^{-k} L\left(\eta^{k}\right)=\sum_{k=1}^{M} \phi^{-k} L\left(\epsilon^{k}\right)
$$

if and only if $\eta^{k}=\epsilon^{k}$ for each $1 \leq k \leq M$.

Example 4. Let $\mathfrak{T}$ be the tiling of $X^{+}$in Example 1. If $x \in \mathbb{Z}[\lambda]$ and $T_{\mathbf{A}}+x \in \mathfrak{T}_{\mathbf{A}}$, then

$$
\phi\left(T_{\mathbf{A}}+x\right)=\left(T_{\mathbf{A}}+\lambda x\right) \cup\left(T_{\mathbf{B}}+1+\lambda x\right) .
$$

Define $\gamma\left(T_{\mathbf{A}}+x\right)=T_{\mathbf{A}}+\lambda x$. If $y \in \mathbb{Z}[\lambda]$ and $T_{\mathbf{B}}+y \in \mathfrak{T}_{\mathbf{B}}$, then

$$
\phi\left(T_{\mathbf{B}}+y\right)=T_{\mathbf{A}}+\lambda y .
$$

Define $\gamma\left(T_{\mathbf{B}}+y\right)=T_{\mathbf{A}}+\lambda y$. It follows that $c\left(T_{\mathbf{A}}+x\right)=x$ and $c\left(T_{\mathbf{B}}+y\right)=y$. So the set of left endpoints of the tiles in $\mathfrak{T}$ form a set of control points. By examining Figure 1 we see that every $x \in T_{\mathbf{A}}$ has a representation of the form

$$
x=\sum_{k=1}^{\infty} \lambda^{-k} L\left(\eta^{k}\right)
$$

where $L\left(\eta^{k}\right) \in\{0,1\}$ and $L\left(\eta^{k}\right)+L\left(\eta^{k+1}\right) \in\{0,1\}$ for all $k \geq 1$.

Definition. A generator for $\mathfrak{T}$ is any tile which contains the origin. 
Theorem 1.5 says that every $x \in X$ may be expressed as the sum of a control point and a series in powers of $\phi$. Let $x \in X$. Since $\phi$ is expansive, there exists $N$ sufficiently large so that $\phi^{-N} x$ belongs to a generator. Suppose $\phi^{-N} x \in T \in \mathfrak{T}_{j}$, for some generator $T$ and $j \in J$. If $c(T)=0$, then there is a path $\left\{\eta^{k}\right\}_{k=1}^{\infty}$ in $\Gamma$ with source $v_{j}$ such that

$$
x=\sum_{k=1}^{\infty} \phi^{N-k} L\left(\eta^{k}\right)
$$

Renumbering the path we have

$$
x=\sum_{k=1-N}^{\infty} \phi^{-k} L\left(\eta^{k}\right) .
$$

We call this expression a digit expansion for $x$ in powers of $\phi$ (with digits labeling a path in $\Gamma$ ). One can check that every $x \in X$ has a digit expansion in powers of $\phi$ if and only if every generator has 0 as its control point. In this case Corollary 1.4 says that distinct generators must have different tile types.

Conversely, suppose that distinct generators have different tile types. We define a tile map which assigns each generator $T$ the unique generator contained in its image $\phi T$. Then the control point for each generator will be 0 and each $x \in X$ will have a digit expansion in powers of $\phi$. We call a tile map which maps generators to generators a generating tile map.

From the above discussion we see that the choice of tile type partition for $\mathfrak{T}$ is not an arbitrary one. If $\mathfrak{T}$ is a subdividing tiling with certain properties, then if we say that $\left\{\mathfrak{T}_{j}\right\}_{j \in J}$ is a tile type partition for $\mathfrak{T}$ we implicitly mean that $\mathfrak{T}$ has those properties with the tile type partition $\left\{\mathfrak{T}_{j}\right\}_{j \in J}$. Not every partition of $\mathfrak{T}$ which is consistent with the definition for $G$-finite tilings will preserve the subdividing properties of $\mathfrak{T}$. Moreover, if we wish to use the tiling to express elements of $X$ using digit expansions, then we must choose a partition which assigns distinct generators different tile types.

One way to construct a new tile type partition for $\mathfrak{T}$ is to perform an insplitting of its subdivision graph. This will define subdivision rules for $\mathfrak{T}$ in terms of a new tile type partition which gives $\mathfrak{T}$ the same subdivision properties as it had with the original partition. Insplitting as a technique used in symbolic dynamics is described in [13]. We give a detailed description of its application to self similar tilings in the Appendix. For now we will illustrate how it works with an example.

Example 5. Let $X=\mathbb{R}^{2}$ and $I^{2}$ be the unit square in $\mathbb{R}^{2}$ with vertices $(0,0)$, $(1,0),(1,1)$, and $(0,1)$. Let

$$
\mathfrak{T}=\left\{I^{2}+z: z \in \mathbb{Z}^{2}\right\}
$$

then $\mathfrak{T}$ is a $\mathbb{Z}^{2}$-finite tiling of $X$. Since every tile in $\mathfrak{T}$ is a $\mathbb{Z}^{2}$-translate of $I^{2}$, we let $\mathfrak{T}$ have one tile type $\mathfrak{T}_{1}$. Let $\phi: \mathbb{R}^{2} \rightarrow \mathbb{R}^{2}$ be dilation by a factor of 2 . We see that

$$
\phi\left(I^{2}\right)=I^{2} \cup\left(I^{2}+(1,0)\right) \cup\left(I^{2}+(1,1)\right) \cup\left(I^{2}+(0,1)\right) .
$$

So $\mathfrak{T}$ is a subdividing tiling with expansion map $\phi$ and tile type partition $\left\{\mathfrak{T}_{1}\right\}$. Define $\gamma: \mathfrak{T} \rightarrow \mathfrak{T}$ by $\gamma\left(I^{2}\right)=I^{2}$. Then for all $z \in \mathbb{Z}^{2}, \gamma\left(I^{2}+z\right)=I^{2}+\phi z$. We find $c\left(I^{2}+z\right)=z$. The subdivision graph $\Gamma$ for $\mathfrak{T}$ is in Figure 2. Each edge $\alpha$ is labeled with $L(\alpha)$ where $L$ is the label map induced by $\gamma$. 


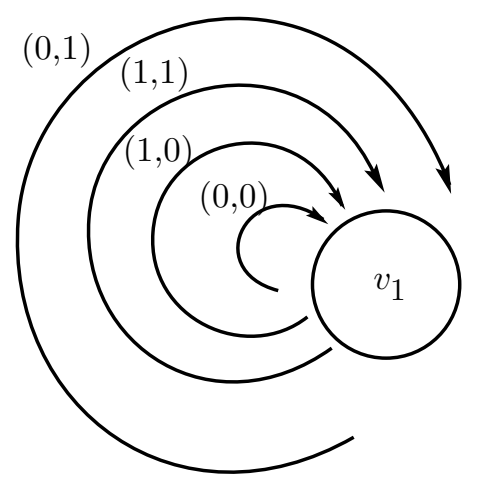

Figure 2. The subdivision graph for the tiling of the plane by unit squares.

Consider the tiling consisting of the tiles $\left\{\phi I^{2}+\phi z: z \in \mathbb{Z}^{2}\right\}$. Each tile $\phi I^{2}+\phi z$ is the union of four tiles of the form $I^{2}+\phi z+(x, y)$ where $(x, y)$ labels an edge of the subdivision graph, $\Gamma$. Let

$$
\hat{\mathfrak{T}}_{1}=\left\{I^{2}+(x, y): x, y \in \mathbb{Z}, \text { and } x+y \text { is even }\right\}
$$

and

$$
\hat{\mathfrak{T}}_{2}=\left\{I^{2}+(x, y): x, y \in \mathbb{Z}, \text { and } x+y \text { is odd }\right\} .
$$

The collection $\left\{\hat{\mathfrak{T}}_{1}, \hat{\mathfrak{T}}_{2}\right\}$ forms a tile type partition for $\mathfrak{T}$ which preserves the subdividing properties of $\mathfrak{T}$. The new subdivision graph for $\mathfrak{T}$ is shown in Figure 3. Note how the edges from the single vertex on the original graph split into two sets and then are copied for each of the two new vertices. This is a single insplitting of the graph. If we color the tiles in $\hat{\mathfrak{T}}_{1}$ white and the tiles in $\hat{\mathfrak{T}}_{2}$ red, then $\mathfrak{T}$ looks like an infinite checkerboard sitting on $\mathbb{R}^{2}$.

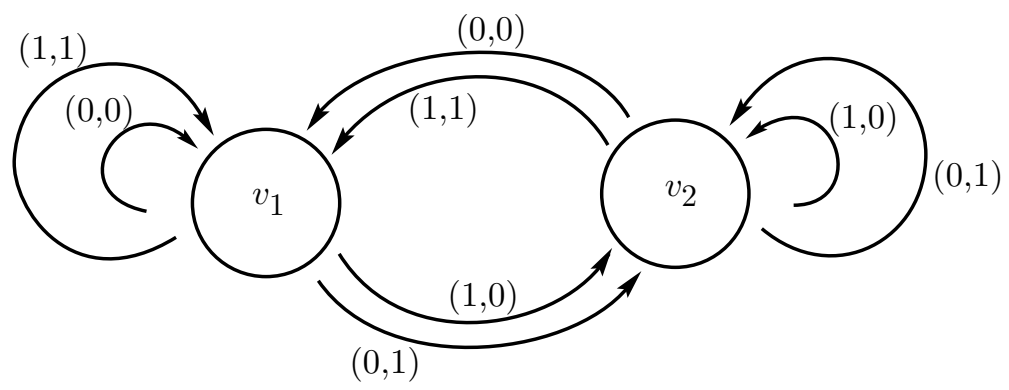

FIGURE 3 . The subdivision graph for the checkerboard tiling of the plane.

Proposition 1.9. Let $j \in J$ and let $T_{1}$ and $T_{2}$ be distinct tiles in $\mathfrak{T}_{j}$. There exists a partition of $\mathfrak{T}_{j}$ into two sets $\hat{\mathfrak{T}}_{j_{1}}$ and $\hat{\mathfrak{T}}_{j_{2}}$ such that

$$
\left\{\mathfrak{T}_{k}: k \in J-\{j\}\right\} \cup\left\{\hat{\mathfrak{T}}_{j_{1}}, \hat{\mathfrak{T}}_{j_{2}}\right\}
$$

is a tile type partition for $\mathfrak{T}$ which preserves the subdividing properties of $\mathfrak{T}$. Moreover $T_{1} \in \hat{\mathfrak{T}}_{j_{1}}$ and $T_{2} \in \hat{\mathfrak{T}}_{j_{2}}$. 
Proof. Please refer to the appendix.

By a finite number of applications of Proposition 1.9 we assume that the tile type partition chosen for $\mathfrak{T}$ assigns distinct generators different tile types and that $\gamma$ is a generating tile map. In particular we index the set of generators with a subset $J_{0} \subset J$. That is, we let $\left\{T_{j}\right\}_{j \in J_{0}}$ be the set of generators for $\mathfrak{T}$, such that $T_{j} \in \mathfrak{T}_{j}$ for each $j \in J_{0}$. It follows immediately from Theorem 1.5 that:

Proposition 1.10. For all $x \in X$ there exists a path $\left\{\eta^{k}\right\}_{k=-N}^{\infty}$ with source in $\left\{v_{j}\right\}_{j \in J_{0}}$ such that $L\left(\eta^{-N}\right) \neq 0$ and

$$
x=\sum_{k=-N}^{\infty} \phi^{-k} L\left(\eta^{k}\right) .
$$

For Lebesgue almost every $x \in X$ there is a unique such path. If $\mathfrak{T}$ has a finite number of local patterns, then for all $x \in X$ there is a uniform bound on the number of such paths which give this representation.

We will refer to this collection of representations as a numeration system for $X$ in powers of $\phi$. Let $c\left(\mathfrak{T}_{j}\right)$ denote the set of control points for tiles in $\mathfrak{T}_{j}$.

Corollary 1.11. For each $j \in J$,

$$
\begin{aligned}
c\left(\mathfrak{T}_{j}\right)= & \left\{\sum_{k=-N}^{0} \phi^{-k} L\left(\eta^{k}\right):\left\{\eta^{k}\right\}_{k=-N}^{0} \text { is a path in } \Gamma\right. \\
& \text { with source in } \left.\left\{v_{k}\right\}_{k \in J_{0}} \text { and target } v_{j}\right\} .
\end{aligned}
$$

Proposition 1.12. If $\mathfrak{T}$ is mixing, then there exists a generating tile map $\gamma$ and $k_{0} \geq 0$ such that, for all $k \geq k_{0}$ and $T \in \mathfrak{T}$, the tile $\gamma^{k}(T)$ has the same tile type as a generator and $c(T) \in G$.

Proof. Suppose that $\mathfrak{T}$ is mixing. Then for each $j \in J$ there exists a minimal $N_{j}$ such that if $T \in \mathfrak{T}$, then $\phi^{N_{j}} T$ contains a tile of the same type as a generator. Let $J_{1} \subset J-J_{0}$ such that for each $j \in J_{1}, N_{j}=1$. Inductively define $J_{M} \subset J-\bigcup_{i=0}^{M-1} J_{i}$ such that for each $j \in J_{M}, N_{j}=M$. Since $J$ is a finite set, the number of nonempty $J_{M}$ is finite. We define a generating tile map so that for each $M$ if $T \in \mathfrak{T}_{j_{1}}$, $j_{1} \in J_{M}$, then $\gamma(T) \in \mathfrak{T}_{j_{2}}, j_{2} \in J_{M-1}$. It follows that, for some $k_{0} \geq 0$ and every $T \in \mathfrak{T}, \gamma^{k}(T), k \geq k_{0}$, is a tile of the same type as some generator $T_{0}$. Moreover $c\left(\gamma^{k}(T)\right)=c\left(\gamma^{k}(T)\right)-c\left(T_{0}\right) \in G$, so $c(T)=\phi^{-k} c\left(\gamma^{k}(T)\right) \in \phi^{-k} G=G$.

\section{PeRiodic tilings of $\mathbb{R}^{n}$}

In this section we construct the $n$-dimensional crinkly tin cans which will ultimately serve as building blocks for Markov partitions. Let $\phi$ be a hyperbolic linear automorphism on $\mathbb{R}^{n}$ with matrix representation in $\mathrm{GL}(n, \mathbb{Z})$ and characteristic polynomial $\chi_{\phi}$ irreducible over $\mathbb{Z}$. The map $\phi$ induces a hyperbolic automorphism $\widehat{\phi}$ on $\mathbb{R}^{n} \bmod \mathbb{Z}^{n}$.

The irreducibility of $\chi_{\phi}$ implies that $\chi_{\phi}$ has no repeated zeroes and hence $\phi$ is diagonalizable. Since $\phi$ is hyperbolic, we may order the eigenvalues for $\phi$ as $\left\{\lambda_{i}\right\}_{i=1}^{n}$ such that

$$
\left|\lambda_{1}\right| \leq\left|\lambda_{2}\right| \leq \cdots\left|\lambda_{l}\right|<1<\left|\lambda_{l+1}\right| \leq \cdots\left|\lambda_{n}\right|
$$


There is a $\phi$-invariant decomposition of $\mathbb{R}^{n}$ into spaces $E_{s}$ and $E_{u}$ such that the eigenvalues for $\left.\phi\right|_{E_{s}}$ are $\left\{\lambda_{i}\right\}_{i=1}^{l}$ and the eigenvalues for $\left.\phi\right|_{E_{u}}$ are $\left\{\lambda_{i}\right\}_{i=l+1}^{n}$. The space $E_{s}$ is the stable eigenspace for $\phi$ and the space $E_{u}$ is the unstable eigenspace for $\phi$. Note that $\left.\phi\right|_{E_{s}} ^{-1}$ and $\left.\phi\right|_{E_{u}}$ are both expansive maps. Hence as in Section 1 we adapt a norm for $\mathbb{R}^{n}$ which reflects the expansive properties of $\left.\phi\right|_{E_{s}} ^{-1}$ and $\left.\phi\right|_{E_{u}}$.

Choose $\varrho$ and $\zeta$ so that

$$
1<\varrho<\min \left\{\left|\lambda_{l}^{-1}\right|,\left|\lambda_{l+1}\right|\right\} \text { and } \zeta>\max \left\{\left|\lambda_{n}\right|,\left|\lambda_{1}^{-1}\right|\right\} .
$$

As in [12] we define a norm $\|\cdot\|$ for $\mathbb{R}^{n}$ such that for each $x \in E_{s}$ we have

$$
\|x\|<\varrho\|x\| \leq\left\|\phi^{-1} x\right\| \leq \zeta\|x\|
$$

and

$$
\zeta^{-1}\|x\| \leq\|\phi x\| \leq \varrho^{-1}\|x\|<\|x\| .
$$

Likewise if $x \in E_{u}$, then

$$
\|x\|<\varrho\|x\| \leq\|\phi x\| \leq \zeta\|x\|
$$

and

$$
\zeta^{-1}\|x\| \leq\left\|\phi^{-1} x\right\| \leq \varrho^{-1}\|x\|<\|x\| .
$$

We give $\mathbb{R}^{n}, E_{s}$, and $E_{u}$ the topologies induced by the norm $\|\cdot\|$.

Since $\chi_{\phi}$ is irreducible over $\mathbb{Z}$, there is no $\phi$-invariant subspace of $\mathbb{R}^{n}$ which intersects $\mathbb{Z}^{n}-\{0\}$. Let $\pi_{s}: \mathbb{R}^{n} \rightarrow E_{s}$ be projection along $E_{u}$ to $E_{s}$. Let $\pi_{u}: \mathbb{R}^{n} \rightarrow$ $E_{u}$ be projection along $E_{s}$ to $E_{u}$. Then $\left.\pi_{s}\right|_{\mathbb{Z}^{n}}$ and $\left.\pi_{u}\right|_{\mathbb{Z}^{n}}$ are bijective $\mathbb{Z}$-linear maps. Let $\zeta_{u}: \pi_{u}\left(\mathbb{Z}^{n}\right) \rightarrow \mathbb{Z}^{n}$ by $\zeta_{u}\left(\pi_{u}(z)\right)=z$. Let $\rho_{s}: \pi_{u}\left(\mathbb{Z}^{n}\right) \rightarrow \pi_{s}\left(\mathbb{Z}^{n}\right)$ by $\rho_{s}\left(\pi_{u}(z)\right)=-\pi_{s}(z)$. We note that $\zeta_{u}$ and $\rho_{s}$ are bijective $\mathbb{Z}$-linear maps which are related by

$$
\rho_{s}(x)=x-\zeta_{u}(x)
$$

for each $x \in \pi_{u}\left(\mathbb{Z}^{n}\right)$.

Let $X_{u}$ be a subset of $E_{u} \cong \mathbb{R}^{n-l}$ which is the closure of its interior. We will make the following assumptions about $X_{u}$.

- $\phi X_{u}=\left.\phi\right|_{E_{u}} X_{u}=X_{u}$.

- $X_{u} \bmod \mathbb{Z}^{n}$ is dense in $\mathbb{R}^{n} \bmod \mathbb{Z}^{n}$.

Suppose that $\mathfrak{T}$ is a $\pi_{u}\left(\mathbb{Z}^{n}\right)$-finite self similar tiling of $X_{u}$ with expansive map $\phi$ and tile type partition $\left\{\mathfrak{T}_{j}\right\}_{j \in J}$. We assume that distinct generators have different tile types. We apply Proposition 1.12 and let $\gamma: \mathfrak{T} \rightarrow \mathfrak{T}$ be a generating tile map so that $c(\mathfrak{T}) \subset \pi_{u}\left(\mathbb{Z}^{n}\right)$. Let $\Gamma$ be the subdivision graph for $\mathfrak{T}$ with vertices indexed by $J$ and edge set $\mathcal{E}$. Let $\left\{T_{j}\right\}_{j \in J_{0}}$ be the set of generators for $\mathfrak{T}$.

If $j \in J_{0}$ and $x \in T_{j}$, then $x$ has a digit expansion $x=\sum_{k=1}^{\infty} \phi^{-k} L\left(\eta^{k}\right)$ for some path $\left\{\eta^{k}\right\}_{k=1}^{\infty}$ with source $v_{j}$. There exists a unique generator $T_{j_{-1}}$ such that $\phi^{-1}(x) \in \phi^{-1}\left(T_{j}\right) \subset T_{j_{-1}}$, and $T_{j_{-1}} \in \mathfrak{T}_{j_{-1}}$. Inductively define for each $N \geq 1$ the generator $T_{j_{-N}} \in \mathfrak{T}_{j_{-N}}$ to be the unique tile containing $\phi^{-N}\left(T_{j}\right)$. For each $k \leq 0$ define

$$
\eta^{k}=\Theta^{-1}\left(v_{j_{k-1}}, v_{j_{k}}, 0\right)
$$


Then $\left\{\eta^{k}\right\}_{k=-\infty}^{\infty}$ is a path in $\Gamma$ for which

$$
x=\sum_{k=-\infty}^{\infty} \phi^{-k} L\left(\eta^{k}\right) .
$$

Note that for every such path $\left\{\eta^{k}\right\}_{k=1}^{\infty}$ the extension $\left\{\eta^{k}\right\}_{k=-\infty}^{\infty}$ is uniquely defined.

Definition. For each $j \in J$ let

$$
\begin{aligned}
S_{j}= & \left\{\eta=\left\{\eta^{k}\right\}_{k \in \mathbb{Z}}: \eta \text { is a path in } \Gamma \text { with } t\left(\eta^{0}\right)=v_{j}\right. \text { and such that there } \\
& \text { exists } \left.N \geq 0 \text { for which } s\left(\eta^{k}\right) \in\left\{v_{j}\right\}_{j \in J_{0}} \text { for all } k \leq-N\right\} .
\end{aligned}
$$

Proposition 2.1. For each $j \in J$ and $x \in T_{j}$ there exists a path $\eta \in S_{j}$ such that $x=\sum_{k \in \mathbb{Z}} \phi^{-k} L\left(\eta^{k}\right)$. For Lebesgue almost every $x$ there is a unique such path. For all $x \in X_{u}$ there is a uniform bound on the number of such paths.

Proof. This follows immediately from Proposition 1.10.

Corollary 2.2. For each $j \in J$

$$
c\left(\mathfrak{T}_{j}\right)=\left\{\sum_{k=-\infty}^{0} \phi^{-k} L\left(\eta^{k}\right):\left\{\eta^{k}\right\}_{k \in \mathbb{Z}} \in S_{j}\right\} .
$$

Definition. For each $j \in J$ let $\Omega_{u, j}$ be the subset of $\mathbb{R}^{n}$ given by

$$
\Omega_{u, j}=\bigcup_{T \in T_{j}}\left(T-\zeta_{u} c(T)\right) .
$$

Since $\rho_{s}(c(T))=c(T)-\zeta_{u} c(T)$, we have

$$
T-\zeta_{u} c(T)=\rho_{s} c(T) \oplus(T-c(T))
$$

where $\rho_{s} c(T) \in E_{s}$ and $T-c(T) \subset E_{u}$. It follows from Corollary 2.2 that

$$
\Omega_{u, j}=\left\{\sum_{k=-\infty}^{0} \phi^{-k} \rho_{s} L\left(\eta^{k}\right)+\sum_{k=1}^{\infty} \phi^{-k} L\left(\eta^{k}\right):\left\{\eta^{k}\right\}_{k \in \mathbb{Z}} \in S_{j}\right\} .
$$

Since $\mathcal{E}$ is finite, if $x \in \Omega_{u, j}$ and $\eta \in S_{j}$ such that

$$
x=\sum_{k=-\infty}^{0} \phi^{-k} \rho_{s} L\left(\eta^{k}\right)+\sum_{k=1}^{\infty} \phi^{-k} L\left(\eta^{k}\right)
$$

then

$$
\begin{aligned}
\|x\| & \leq \sum_{k=-\infty}^{0} \varrho^{k}\left\|\rho_{s} L\left(\eta^{k}\right)\right\|+\sum_{k=1}^{\infty} \varrho^{-k}\left\|L\left(\eta^{k}\right)\right\| \\
& \leq \frac{2}{1+\varrho^{-1}} \max \left\{\left\|\rho_{s} L(\alpha)\right\|,\|L(\alpha)\|: \alpha \in \mathcal{E}\right\} .
\end{aligned}
$$

So $\Omega_{u, j}$ is bounded.

Definition. $\quad$ For each $j \in J$, let $\Omega_{j}=\operatorname{Clos}\left(\Omega_{u, j}\right)$.

- Let $\Omega_{u}=\bigcup_{j \in J} \Omega_{u, j}$.

- Let $\Omega=\operatorname{Clos}\left(\Omega_{u}\right)$. 
Since $\Omega_{u}$ is bounded, $\Omega$ is a compact set. Since $X_{u} \bmod \mathbb{Z}^{n}=\Omega_{u} \bmod \mathbb{Z}^{n}$ and $X_{u} \bmod \mathbb{Z}^{n}$ is dense in $\mathbb{R}^{n} \bmod \mathbb{Z}^{n}$, we have that

$$
\Omega \bmod \mathbb{Z}^{n}=\mathbb{R}^{n} \bmod \mathbb{Z}^{n} .
$$

Hence

$$
\bigcup_{z \in \mathbb{Z}^{n}}(\Omega+z)=\mathbb{R}^{n}
$$

It turns out that under special circumstances the collection

$$
\left\{\Omega+z: z \in \mathbb{Z}^{n}\right\}
$$

forms a periodic tiling of $\mathbb{R}^{n}$. This is the first step in constructing a Markov partition for $\phi \bmod \mathbb{Z}^{n}$.

We begin by taking advantage of a lot of foreknowledge and discuss the shift of finite type which will eventually represent the hyperbolic toral automorphism induced by $\phi$.

Definition. If $\Gamma$ is a graph with edge set $\mathcal{E}$, then the graph shift $\Sigma_{\Gamma}$ is the shift of finite type over the alphabet $\mathcal{E}$ specified by

$$
\Sigma_{\Gamma}=\left\{\eta=\left\{\eta^{k}\right\}_{k \in \mathbb{Z}} \in \mathcal{E}^{\mathbb{Z}}: \eta \text { is a path in } \Gamma\right\} .
$$

The shift operator, $\sigma_{\Gamma}: \Sigma_{\Gamma} \rightarrow \Sigma_{\Gamma}$, is defined for each $\eta \in \Sigma_{\Gamma}$ and $k \in \mathbb{Z}$ so that

$$
\sigma_{\Gamma} \eta^{k}=\eta^{k+1}
$$

We define a metric $d_{\Gamma}$ on $\Sigma_{\Gamma}$ such that if $\eta, \epsilon \in \Sigma_{\Gamma}$ and $k \in \mathbb{Z}$, then

$$
d_{\Gamma}(\eta, \epsilon)=\frac{1}{1+|k|}
$$

if $|k|$ is minimal such that $\eta^{k} \neq \epsilon^{k}$. We give $\Sigma_{\Gamma}$ the topology induced by the metric $d_{\Gamma}$. In this topology $\Sigma_{\Gamma}$ is compact and $\sigma_{\Gamma}$ is a homeomorphism.

For each $\alpha \in \mathcal{E}$ and $i \in \mathbb{Z}$ define

$$
C_{i}(\alpha)=\left\{\eta \in \Sigma_{\Gamma}: \eta^{i}=\alpha\right\} .
$$

Note that $C_{i}(\alpha)=\sigma_{\Gamma}^{-i} C_{0}(\alpha)$. For each finite path $\left\{\eta^{k}\right\}_{k=-N}^{M}$ in $\Gamma$ define

$$
C_{0}\left(\eta^{-N} \eta^{-N+1} \ldots \eta^{M}\right)=\bigcap_{k=0}^{N+M} C_{k}\left(\eta^{-N+k}\right)
$$

and for each $i \in \mathbb{Z}$

$$
C_{i}\left(\eta^{-N} \eta^{-N+1} \cdots \eta^{M}\right)=\sigma_{\Gamma}^{-i} C_{0}\left(\eta^{-N} \eta^{-N+1} \cdots \eta^{M}\right) .
$$

We call the set $C_{i}\left(\eta^{-N} \ldots \eta^{M}\right)$ a cylinder set in $\Sigma_{\Gamma}$. The cylinder sets are both open and compact in the topology of $\Sigma_{\Gamma}$.

Let $\Sigma_{\Gamma}$ be the graph shift induced by the subdivision graph $\Gamma$ for $\mathfrak{T}$. For each $j \in J$ let

Note that

$$
\bar{S}_{j}=\left\{\eta \in \Sigma_{\Gamma}: t\left(\eta^{0}\right)=v_{j}\right\}
$$

$$
\Sigma_{\Gamma}=\bigcup_{j \in J} \bar{S}_{j}
$$

where this is a disjoint union and $\bar{S}_{j}$ is compact in $\Sigma_{\Gamma}$. 
Definition. Define $\psi: \Sigma_{\Gamma} \rightarrow \mathbb{R}^{n}$ for $\eta \in \Sigma_{\Gamma}$ by

$$
\psi(\eta)=\sum_{k=-\infty}^{0} \phi^{-k} \rho_{s} L\left(\eta^{k}\right)+\sum_{k=1}^{\infty} \phi^{-k} L\left(\eta^{k}\right) .
$$

One can check that $\psi$ is well defined for each $\eta \in \Sigma_{\Gamma}$.

Suppose $\eta$ and $\epsilon$ are elements of $\Sigma_{\Gamma}$ and $N \in \mathbb{Z}$ such that

$$
d_{\Gamma}(\eta, \epsilon) \leq \frac{1}{1+|N|}
$$

then

$$
\begin{aligned}
\|\psi(\eta)-\psi(\epsilon)\| & \leq \sum_{k=-\infty}^{-N}\left\|\phi^{-k} \rho_{s}\left(L\left(\eta^{k}\right)-L\left(\epsilon^{k}\right)\right)\right\|+\sum_{k=N}^{\infty}\left\|\phi^{-k}\left(L\left(\eta^{k}\right)-L\left(\epsilon^{k}\right)\right)\right\| \\
& \leq \frac{2 \varrho^{-N}}{1+\varrho^{-1}} \max \left\{\left\|\rho_{s} L(\alpha)\right\|,\|L(\alpha)\|: \alpha \in \mathcal{E}\right\} .
\end{aligned}
$$

Hence $\psi$ is a uniformly continuous map from $\Sigma_{\Gamma}$ to $\mathbb{R}^{n}$. Note that $\psi\left(S_{j}\right)=\Omega_{u, j}$.

Proposition 2.3. For each $j \in J, S_{j}$ is dense in $\bar{S}_{j}$ and $\psi\left(\bar{S}_{j}\right)=\Omega_{j}$.

Proof. Let $j \in J$ and $\eta \in \bar{S}_{j}$. For each $k \in \mathbb{Z}$ suppose

$$
\Theta\left(\eta^{k}\right)=\left(v_{j_{k-1}}, v_{j_{k}}, L\left(\eta^{k}\right)\right)
$$

For each $N>0$ fix $\epsilon_{N} \in S_{j_{-N}}$ and for each $k \in \mathbb{Z}$ define $\eta_{N}^{k-N}=\epsilon_{N}^{k}$ for $k \leq 0$, and $\eta_{N}^{k-N}=\eta^{k-N}$ for $k>0$. Then $\eta_{N}=\left\{\eta_{N}^{k}\right\}_{k \in \mathbb{Z}} \in S_{j}$ and

$$
d_{\Gamma}\left(\eta_{N}, \eta\right) \leq \frac{1}{1+N}
$$

It follows that $\bar{S}_{j}=\operatorname{Clos}\left(S_{j}\right)$.

Since $\bar{S}_{j}$ is compact in $\Sigma_{\Gamma}$ and $\psi$ is continuous, $\psi\left(\bar{S}_{j}\right)$ is a compact subset of $\mathbb{R}^{n}$. Since $S_{j}$ is dense in $\bar{S}_{j}, \Omega_{u, j}=\psi\left(S_{j}\right)$ is dense in $\psi\left(\bar{S}_{j}\right)$. Hence $\Omega_{j}=\psi\left(\bar{S}_{j}\right)$.

Corollary 2.4. The set $\bigcup_{j \in J} S_{j}$ is dense in $\Sigma_{\Gamma}$ and

$$
\psi\left(\Sigma_{\Gamma}\right)=\Omega \text {. }
$$

Proposition 2.5. For all $\eta \in \Sigma_{\Gamma}$ and $N \geq 0$ we have

$$
\psi\left(\sigma_{\Gamma}^{N} \eta\right)=\phi^{N} \psi(\eta)-\phi^{N} \sum_{k=1}^{N} \phi^{-k} \zeta_{u} L\left(\eta^{k}\right)
$$

and

$$
\psi\left(\sigma_{\Gamma}^{-N} \eta\right)=\phi^{-N} \psi(\eta)+\phi^{-N} \sum_{k=1-N}^{0} \phi^{-k} \zeta_{u} L\left(\eta^{k}\right) .
$$

Proof. This follows from the definitions and lots of reindexing.

Proposition 2.6. (1) For each $\eta \in \bar{S}_{j}$ there exists $\bar{\eta} \in \bar{S}_{j}$ such that $\pi_{s} \psi(\eta)=$ $\psi(\bar{\eta}) \in \Omega_{j} \cap E_{s}$ and $L\left(\bar{\eta}^{k}\right)=0$ for $k \geq 1$.

(2) $\phi \pi_{s} \Omega \subset \pi_{s} \Omega=\Omega \cap E_{s}$.

Proof. To prove (1) just note that every vertex is the source of a path labeled only with 0. Part (2) follows from (1). 
Proposition 2.7. The map $\psi$ is boundedly finite to one.

Proof. Let $x \in \Omega$ and $\left\{\eta_{i}\right\}_{i \in I} \subset \psi^{-1}(x)$ for some finite indexing set $I$. Without loss of generality we may assume that the paths $\eta_{i}$ and $\eta_{i^{\prime}}$ are distinct for each pair of distinct $i, i^{\prime} \in I$. Hence there exists $N \geq 0$ such that the paths

$$
\left\{\eta_{i}^{k}\right\}_{k=-N}^{\infty} \text { and }\left\{\eta_{i^{\prime}}^{k}\right\}_{k=-N}^{\infty}
$$

are distinct for all $i \neq i^{\prime}$.

Since $\Omega$ is compact in $\mathbb{R}^{n}$, there exists a finite collection of $z \in \mathbb{Z}^{n}$ such that

$$
\Omega \cap(\Omega+z) \neq \emptyset .
$$

Hence there exists a bound $b_{1}$ such that for all $y \in \Omega$ the number of points in $y+\mathbb{Z}^{n}$ belonging to $\Omega$ is less than or equal to $b_{1}$. By Proposition 2.5 for each $i \in I$

$$
\phi^{-N-1} x+\phi^{-N-1} \sum_{k=-N}^{0} \phi^{-k} \zeta_{u} L\left(\eta_{i}^{k}\right)=\psi\left(\sigma_{\Gamma}{ }^{-N-1} \eta_{i}\right) \in \Omega .
$$

So for each $i \neq i^{\prime}$ in $I$ there exists $z \in \mathbb{Z}^{n}$ such that

$$
\psi\left(\sigma_{\Gamma}{ }^{-N-1} \eta_{i}\right)=\psi\left(\sigma_{\Gamma}{ }^{-N-1} \eta_{i^{\prime}}\right)+z .
$$

Hence the number of distinct points in $\left\{\psi\left(\sigma_{\Gamma}{ }^{-N-1} \eta_{i}\right)\right\}_{i \in I}$ is less than or equal to $b_{1}$. Let $y \in\left\{\psi\left(\sigma_{\Gamma}^{N-1} \eta_{i}\right)\right\}_{i \in I}$. Then for some $i$

$$
\pi_{u}(y)=\sum_{k=1}^{\infty} \phi^{-k} L\left(\sigma_{\Gamma}^{-N-1} \eta_{i}^{k}\right)
$$

By Corollary 1.7 there is a bound $\bar{b}$ on the number of paths which give a representation for $\pi_{u}(y)$. Hence the number of elements of $\left\{\sigma_{\Gamma}{ }^{-N-1} \eta_{i}\right\}_{i \in I}$ which are mapped by $\psi$ to the same point is less than or equal to $\bar{b}$. It follows that $|I| \leq b_{1} \bar{b}$. Since $\left\{\eta_{i}\right\}_{i \in I}$ was an arbitrary finite subset of $\psi^{-1}(x)$ and since $x$ was arbitrary in $\Omega$, we have that $\left|\psi^{-1}(x)\right| \leq b_{1} \bar{b}$ for all $x \in \Omega$.

If $\left\{\Omega+z: z \in \mathbb{Z}^{n}\right\}$ is a tiling of $\mathbb{R}^{n}$, then we will think of $\Omega$ as representing the $n$-dimensional torus. We let $\Pi$ be the quotient map from $\mathbb{R}^{n}$ to $\mathbb{R}^{n} \bmod \mathbb{Z}^{n}$ and study the map

$$
\Pi \circ \psi:\left(\Sigma_{\Gamma}, \sigma_{\Gamma}\right) \rightarrow(\Pi \Omega, \Pi \circ \phi) .
$$

The goal of the remainder of this section is to demonstrate what is needed to check in order to determine if $\left\{\Omega+z: z \in \mathbb{Z}^{n}\right\}$ is indeed a tiling of $\mathbb{R}^{n}$.

Definition. A finite collection of compact sets $\left\{C_{1}, C_{2}, \ldots, C_{k}\right\}$ in $\mathbb{R}^{n}$ induces a periodic tiling of $\mathbb{R}^{n} \bmod \mathbb{Z}^{n}$ if

(1) $\left(\bigcup_{i=1}^{k} C_{i}\right) \bmod \mathbb{Z}^{n}=\mathbb{R}^{n} \bmod \mathbb{Z}^{n}$,

(2) each $C_{i}$ is the closure of its interior, and

(3) for all $z \in \mathbb{Z}^{n}$ if

$$
\left(C_{i}+z\right) \cap \operatorname{Int}\left(C_{j}\right) \neq \emptyset,
$$

then $z=0$ and $i=j$.

Proposition 2.8. (1) For each $j \in J, \pi_{s}\left(\Omega_{j}\right)$ is the closure of its interior.

(2) For each $j \in J, \Omega_{j}$ is the closure of its interior.

(3) $\Omega$ is the closure of its interior and $\pi_{s}(\Omega)$ is the closure of its interior. 
Proof. Since

$$
\bigcup_{z \in \mathbb{Z}^{n}}\left(\cup_{j \in J}\left(\Omega_{j}+z\right)\right)=\mathbb{R}^{n}
$$

we apply the Baire category theorem. That is, for some $j \in J$ we have that $\Omega_{j}$ has non-empty interior. But

$$
\Omega_{j}=\pi_{s}\left(\Omega_{j}\right) \oplus(T-c(T))
$$

for any $T \in \mathfrak{T}_{j}$, so $\Omega_{j}$ has non-empty interior if and only if $\pi_{s}\left(\Omega_{j}\right)$ has non-empty interior. It follows that by showing (1) we immediately get (2). For each $j^{\prime} \in J$, let $U_{j^{\prime}}=\operatorname{Int}\left(\pi_{s} \Omega_{j^{\prime}}\right)$. By the above remarks $U_{j} \neq \emptyset$. By Proposition 2.6 for each $x \in U_{j}$ there exists $\eta_{x} \in \bar{S}_{j}$ such that $\psi\left(\eta_{x}\right)=x$ and $L\left(\eta_{x}^{k}\right)=0$ for $k \geq 1$. Since $\mathfrak{T}$ is mixing, for each $j^{\prime} \in J$ there exists a path $\left\{\epsilon^{k}\right\}_{k=1}^{N}$ in $\Gamma$ such that $s\left(\epsilon^{1}\right)=j$ and $t\left(\epsilon^{N}\right)=j^{\prime}$. Let $\bar{\epsilon} \in \bar{S}_{j}$ be defined so that

$$
\begin{aligned}
\bar{\epsilon}^{k}=\eta_{x}^{k} & \text { for } k \leq 0, \\
\bar{\epsilon}^{k}=\epsilon^{k} & \text { for } 1 \leq k \leq N, \text { and } \\
L\left(\bar{\epsilon}^{k}\right)=0 & \text { for } k>N .
\end{aligned}
$$

Then

$$
\begin{aligned}
\phi^{N}(\psi(\bar{\epsilon}))-\phi^{N} \sum_{k=1}^{N} \phi^{-k} \zeta_{u} L\left(\bar{\epsilon}^{k}\right) & =\psi\left(\sigma_{\Gamma}^{N} \bar{\epsilon}\right) \\
& =\sum_{k=-\infty}^{0} \phi^{-k} \rho_{s} L\left(\bar{\epsilon}^{k+N}\right)+\sum_{k=1}^{\infty} \phi^{-k} L\left(\bar{\epsilon}^{k+N}\right) \\
& =\sum_{k=-\infty}^{-N} \phi^{-k} \rho_{s} L\left(\eta_{x}^{k+N}\right)+\sum_{k=1-N}^{0} \phi^{-k} \rho_{s} L\left(\epsilon^{k+N}\right) \\
& =\phi^{N} \psi\left(\eta_{x}\right)+\sum_{k=1-N}^{0} \phi^{-k} \rho_{s} L\left(\epsilon^{k+N}\right) .
\end{aligned}
$$

Since $t\left(\epsilon^{N}\right)=v_{j^{\prime}}$, we have $\psi\left(\sigma_{\Gamma}^{N} \bar{\epsilon}\right) \in \pi_{s} \Omega_{j^{\prime}}$. Since $x$ was arbitrary in $U_{j}$,

$$
\phi^{N} U_{j}+\sum_{k=1-N}^{0} \phi^{-k} \rho_{s} L\left(\epsilon^{k+N}\right) \subset U_{j^{\prime}} .
$$

So $U_{j^{\prime}} \neq \emptyset$ for all $j^{\prime} \in J$.

Let $T_{j_{0}}$ be a generator for $\mathfrak{T}$. Then there is a unique generator $T_{j_{1}}$ for $\mathfrak{T}$ such that

$$
T_{j_{0}}=\gamma\left(T_{j_{1}}\right)
$$

For each $N$ let $T_{j_{N}}$ be the unique generator for $\mathfrak{T}$ such that

$$
T_{j_{0}}=\gamma^{N}\left(T_{j_{N}}\right) .
$$

Then for $T \in \mathfrak{T}_{j_{N}}$, we have that $\phi^{N} c(T)=c\left(\gamma^{N} T\right) \in c\left(\mathfrak{T}_{j_{0}}\right)$. Hence

$$
\bigcup_{N=0}^{\infty} \phi^{N} U_{j_{N}} \subset U_{j_{0}}
$$


Since $\left.\phi\right|_{E_{s}} ^{-1}$ is expansive, 0 belongs to the closure of $U_{j_{0}}$. Since $T_{j_{0}}$ was an arbitrary generator for $\mathfrak{T}$, for all generators $T_{j} \in \mathfrak{T}_{j}$, we have that 0 belongs to the closure of $U_{j}$.

Let $j \in J$ and $T \in \mathfrak{T}_{j}$. Then for some generator $T_{j_{0}}$ there exists an $N \geq 0$ such that $T \subset \phi^{N} T_{j_{0}}$ in which case

$$
c(T)=\phi^{N} c\left(T_{j_{0}}\right)+c(T) .
$$

So for all $T^{\prime} \in \mathfrak{T}_{j_{0}}$,

$$
\phi^{N} c\left(T^{\prime}\right)+c(T) \in c\left(\mathfrak{T}_{j}\right)
$$

Hence $\phi^{N} U_{j_{0}}+\rho_{s} c(T) \subset U_{j}$. Since 0 belongs to the closure of $\phi^{N} U_{j_{0}}$ we have that $\rho_{s} c(T)$ belongs to the closure of $U_{j}$. But $T$ was arbitrary in $\mathfrak{T}_{j}$ and

$$
\pi_{s} \Omega_{j}=\operatorname{Clos}\left\{\rho_{s} c(T): T \in \mathfrak{T}_{j}\right\} .
$$

Hence $\pi_{s} \Omega_{j} \subset \operatorname{Clos}\left(U_{j}\right)$. This proves (1) and (2).

Finally we note that $\Omega=\bigcup_{j \in J} \Omega_{j}$ and $\pi_{s} \Omega=\bigcup_{j \in J} \pi_{s} \Omega_{j}$. Since both unions are finite, we obtain (3).

Theorem 2.9. The collection $\left\{\Omega_{j}\right\}_{j \in J}$ induces a periodic tiling of $\mathbb{R}^{n} \bmod \mathbb{Z}^{n}$ if and only if for all $z \in \mathbb{Z}^{n}$ and $j \in J$ such that $X_{u}-z \cap \operatorname{Int}\left(\Omega_{j}\right) \neq \emptyset$ we have that $z \in \zeta_{u} c\left(\mathfrak{T}_{j}\right)$.

Proof. Suppose the sets $\Omega_{j}$ do induce a periodic tiling of $\mathbb{R}^{n} \bmod \mathbb{Z}^{n}$. Let $z \in \mathbb{Z}^{n}$ and suppose $X_{u}-z \cap \operatorname{Int}\left(\Omega_{j}\right) \neq \emptyset$ for some $j \in J$. Then for some $j^{\prime} \in J$ and $T$ in $\mathfrak{T}_{j^{\prime}}$ we have that

$$
(T-z) \cap \operatorname{Int}\left(\Omega_{j}\right) \neq \emptyset .
$$

Since $T \in \mathfrak{T}_{j^{\prime}}$, we have that

$$
\left(T-\zeta_{u} c(T)\right) \subset \Omega_{j^{\prime}}
$$

Moreover,

$$
\left(T-\zeta_{u} c(T)+\left(\zeta_{u} c(T)-z\right)\right) \cap \operatorname{Int}\left(\Omega_{j}\right) \neq \emptyset
$$

so

$$
\left(\Omega_{j^{\prime}}+\left(\zeta_{u} c(T)-z\right)\right) \cap \operatorname{Int}\left(\Omega_{j}\right) \neq \emptyset .
$$

By our hypothesis we have $\zeta_{u} c(T)=z$ and $j=j^{\prime}$. Hence $z \in \zeta_{u} c\left(\mathfrak{T}_{j}\right)$.

Conversely, suppose $X_{u}-z \cap \operatorname{Int}\left(\Omega_{j}\right) \neq \emptyset$ implies that $z \in \zeta_{u} c\left(\mathfrak{T}_{j}\right)$. Let $z \in \mathbb{Z}^{n}$ and $j, k \in J$ and suppose that

$$
\left(\Omega_{j}+z\right) \cap \operatorname{Int}\left(\Omega_{k}\right) \neq \emptyset .
$$

Since $\Omega_{u, j}$ is dense in $\Omega_{j}$ for some $T \in \mathfrak{T}_{j}$, we have that

$$
\left(T-\zeta_{u} c(T)+z\right) \cap \operatorname{Int}\left(\Omega_{k}\right) \neq \emptyset .
$$

Since $T$ is the closure of its interior, we have

$$
\left(\operatorname{Int}(T)-\zeta_{u} c(T)+z\right) \cap \operatorname{Int}\left(\Omega_{k}\right) \neq \emptyset .
$$

By the hypothesis $\zeta_{u} c(T)-z \in \zeta_{u} c\left(\mathfrak{T}_{k}\right)$. Hence for some $T^{\prime} \in \mathfrak{T}_{k}$ we have

$$
\zeta_{u} c\left(T^{\prime}\right)=\zeta_{u} c(T)-z \text {. }
$$

Moreover,

$$
\left(\operatorname{Int}(T)-\zeta_{u} c(T)+z\right) \cap\left(T^{\prime}-\zeta_{u} c\left(T^{\prime}\right)\right) \neq \emptyset .
$$


Hence $\operatorname{Int}(T) \cap T^{\prime} \neq \emptyset$. So $T=T^{\prime}$ and $\zeta_{u} c(T)=\zeta_{u} c\left(T^{\prime}\right)$. It follows that $j=k$ and $z=0$.

In general Theorem 2.9 may or may not be useful to check if we have a periodic tiling of $\mathbb{R}^{n} \bmod \mathbb{Z}^{n}$. The set $X_{u}$ may not be connected so just checking if

$$
X_{u}-z \cap \operatorname{Int}\left(\Omega_{j}\right) \neq \emptyset
$$

may be difficult. Fortunately, the tilings that we will be interested in have a bit more structure.

Definition. If $\left\{\Omega_{j}\right\}_{j \in J}$ yields a periodic tiling of $\mathbb{R}^{n} \bmod \mathbb{Z}^{n}$, then we will call $\mathfrak{T}$ a Markov tiling.

The self similar tilings that we will be using to construct Markov partitions will be Markov tilings. In general the tiled space $X_{u}$ will be a semigroup. When $X_{u}$ is a semigroup, we can determine if $\left\{\Omega_{j}\right\}_{j \in J}$ induces a periodic tiling by considering the lattice points in $\mathbb{Z}^{n}$ which are close to $X_{u}$.

Definition. Let $P_{0}$ be the union of the generators for $\mathfrak{T}$. Let $\left[P_{0}\right]$ be the set of $\pi_{u}\left(\mathbb{Z}^{n}\right)$-translates of $P_{0}$ which have the same pattern as $P_{0}$.

If $P_{0}+g \in\left[P_{0}\right]$, then each tile in $P_{0}+g$ is of the same type as a generator. Moreover each tile in $P_{0}+g$ has $g$ as its control point. For each $P \in\left[P_{0}\right]$ let $c(P)$ denote this control point, so that $P_{0}+c(P)=P$. Let $c\left[P_{0}\right]$ denote the set of all such control points. It turns out that if the elements of $\mathbb{Z}^{n}$ which project onto $c\left[P_{0}\right]$ include all the elements of $\mathbb{Z}^{n}$ within a certain bounded region of $X_{u}$, then we have a periodic tiling.

Definition. Let

$$
X_{s}=\bigcup_{k=0}^{\infty} \phi^{-k} \pi_{s} \Omega
$$

Since $\pi_{s} \Omega=\left\{\sum_{k=-\infty}^{0} \phi^{-k} \rho_{s} L\left(\eta^{k}\right): \eta \in \Sigma_{\Gamma}\right\}$, we have

$$
X_{s}=\left\{\sum_{k=-\infty}^{M} \phi^{-k} \rho_{s} L\left(\eta^{k}\right): \eta \in \Sigma_{\Gamma}, M \in \mathbb{Z}\right\} .
$$

Note that $\phi X_{s}=X_{s}$ and $X_{s}$ is the closure of its interior.

Lemma 2.10. For every $M \in \mathbb{Z}$ there exists $N \in \mathbb{Z}$ such that if $\eta \in \Sigma_{\Gamma}, M^{\prime} \in \mathbb{Z}$, and

$$
\sum_{k=-\infty}^{M^{\prime}} \phi^{-k} \rho_{s} L\left(\eta^{k}\right) \in \phi^{N} \pi_{s} \Omega
$$

then $M^{\prime} \leq M$.

Proof. Let $\eta \in \Sigma_{\Gamma}$ and $M^{\prime} \in \mathbb{Z}$. Suppose

$$
x=\sum_{k=-\infty}^{M^{\prime}} \phi^{-k} \rho_{s} L\left(\eta^{k}\right) \in \pi_{s} \Omega .
$$


We wish to find a bound $M$ independent of $x$ such that $M^{\prime} \leq M$. Clearly $M \geq 0$. Assume that $M^{\prime}>0$ and let $z=\sum_{k=1}^{M^{\prime}} \phi^{-k} \zeta_{u} L\left(\eta^{k}\right)$, then

$$
x=\sum_{k=-\infty}^{0} \phi^{-k} \rho_{s} L\left(\eta^{k}\right)+\sum_{k=1}^{M^{\prime}} \phi^{-k} \rho_{s} L\left(\eta^{k}\right) \in \Omega-z .
$$

By an application of Corollary 1.10 there is a bound on the number of finite paths in $\Gamma$ given by $\left\{\eta^{k}\right\}_{k \in I}$ such that $\pi_{u}(z)=\sum_{k \in I} \phi^{-k} L\left(\eta^{k}\right)$. Let $M_{z}=\max _{I}\{i \in I\}$. Since $\Omega$ is compact, there are at most a finite number of lattice points $z \in \mathbb{Z}^{n}$ such that

$$
(\Omega-z) \cap \pi_{s} \Omega \neq \emptyset .
$$

Let $M_{0}=\max \left\{M_{z}: z \in \mathbb{Z}^{n},(\Omega-z) \cap \pi_{s} \Omega \neq \emptyset\right\}$; then $M^{\prime} \leq M_{0}$ and $M_{0}$ is independent of $x$. We find for all $N \in \mathbb{Z}$ if $x \in \phi^{N} \pi_{s} \Omega$, then $M^{\prime} \leq M_{0}-N$. Let $N=M_{0}-M$.

Lemma 2.11. For each $N \geq 0, \phi^{N} c\left[P_{0}\right] \subset c\left[P_{0}\right]$.

Theorem 2.12. Suppose that $X_{u}$ is a semigroup and for all

$$
z \in-X_{s} \oplus X_{u} \cap \mathbb{Z}^{n}
$$

there exists $N \geq 0$ such that $\phi^{N} z \in \zeta_{u} c\left[P_{0}\right]$. Then $\mathfrak{T}$ is a Markov tiling.

What this theorem essentially says is that if every translate of $\Omega$ by a lattice point in $\mathbb{Z}^{n}$ which is close to $X_{u}$ intersects $X_{u}$ in a set with the same pattern as $P_{0}$, then we have a periodic tiling of $\mathbb{R}^{n} \bmod \mathbb{Z}^{n}$. It turns out that many tilings are constructed with this property built in. So checking if we have a Markov tiling becomes trivial. We will prove Theorem 2.12 at the end of this section.

Corollary 2.13. Suppose that $X_{u}$ is a semigroup and $\mathfrak{T}$ has only one generator. If for all $z \in-X_{s} \oplus X_{u} \cap \mathbb{Z}^{n}$ we have $z \in \bigcup_{k=0}^{\infty} \phi^{-k} \zeta_{u} c(\mathfrak{T})$, then $\mathfrak{T}$ is a Markov tiling.

Good examples of Markov tilings may be found by studying the $\beta$-shift, for $\beta$ a Pisot number. We say that $\beta$ is a Pisot number if $\beta$ is an algebraic integer greater than 1 with Galois conjugates all having modulus less than 1 . It is well known that there is a one-sided shift space which behaves like multiplication times $\beta \bmod 1$. Define $T_{\beta}:[0,1) \rightarrow[0,1)$ by

$$
T_{\beta}(x)=\beta x-[\beta x]
$$

where $[\beta x]$ denotes the greatest integer less than or equal to $\beta x$. For each $x \in[0,1)$ there is a well defined digit representation

$$
x=\sum_{k=1}^{\infty} \beta^{-k} b_{k}
$$

where

$$
b_{k}=\left[\beta T_{\beta}^{k-1} x\right] .
$$

We call this the $\beta$-expansion of $x$. Moreover there is a sequence denoted by $\operatorname{carry}(\beta)=a_{1} a_{2} a_{3} \ldots$ such that for all $x$ the corresponding sequence $b_{1} b_{2} b_{3} \ldots$ is lexicographically less than $a_{1} a_{2} a_{3} \ldots$. We say $\left\{b_{k}\right\}_{k=1}^{\infty}$ is lexicographically less than $\left\{a_{k}\right\}_{k=1}^{\infty}$ if for some $N \geq 0$ we have $b_{k}=a_{k}$ for all $k<N$ and $b_{N}<a_{N}$. (If 
$N=0$, then $b_{1}<a_{1}$.) We call carry $(\beta)$ the carry sequence for $\beta$ as in [17] and note that $\operatorname{carry}(\beta)$ works for numbers in base $\beta$ just as the sequence $999999 \cdots$ works for numbers in base 10. In particular

$$
1=\sum_{k=1}^{\infty} \beta^{-k} a_{k}
$$

To find $\operatorname{carry}(\beta)$ we let $a_{1}$ be the greatest integer strictly less than $\beta$. Let $a_{2}$ be the greatest integer strictly less than $\beta\left(\beta-a_{1}\right)$. Let $a_{i}$ be the greatest integer strictly less than $\beta^{i}-\sum_{k=1}^{i-1} \beta^{i-k} a_{k}$. Since $\beta$ is $\operatorname{Pisot}, \operatorname{carry}(\beta)$ is an eventually repeating sequence which has an infinite number of non-zero entries.

The $\beta$-shift $\Sigma_{\beta}$ is the set of sequences in $\{0,1, \ldots,[\beta]\}$ which are lexicographically less than or equal to $\operatorname{carry}(\beta)$. The shift operator is the one-sided shift $\sigma_{\beta}$ such that

$$
\sigma_{\beta}\left(b_{1} b_{2} b_{3} \ldots\right)=b_{2} b_{3} b_{4} \ldots
$$

Let $x \in[0,1)$ and let $\left\{b_{k}\right\}_{k=1}^{\infty} \in \Sigma_{\beta}$ such that

$$
x=\sum_{k=1}^{\infty} \beta^{-k} b_{k} .
$$

Then

$$
\beta x=b_{1}+\sum_{k=1}^{\infty} \beta^{-k} b_{k+1} .
$$

If $\left\{b_{k+1}\right\}_{k=1}^{\infty}$ is lexicographically less than $\operatorname{carry}(\beta)$, then we have

$$
T_{\beta} x=\sum_{k=1}^{\infty} \beta^{-k} b_{k+1} .
$$

Otherwise we have $\sum_{k=1}^{\infty} \beta^{-k} b_{k+1}=1$ and $\beta x=b_{1}+1$. In this case $T_{\beta} x=0$.

Suppose $\beta$ is a Pisot unit. That is, the product of $\beta$ and its Galois conjugates is 1. Suppose $\mathbb{Z}[\beta]$ has dimension $n$. Let $\phi \in \operatorname{GL}(n, \mathbb{Z})$ be the companion matrix with characteristic polynomial equal to the minimal polynomial for $\beta$. Then $\phi$ induces a hyperbolic automorphism of $\mathbb{R}^{n} \bmod \mathbb{Z}^{n}$. Let $e$ be a unit vector in $\mathbb{Z}^{n}$. Then $\mathbb{Z}[\phi] e=\mathbb{Z}^{n}$. Let $e_{u}=\pi_{u} e$; then $E_{u}=\mathbb{R} e_{u}$. We construct a self similar tiling $\mathfrak{T}$ of $X_{u}=[0, \infty) e_{u}$. If

$$
\operatorname{carry}(\beta)=a_{1} a_{2} \ldots a_{q}\left(a_{q+1} \ldots a_{q+p}\right)
$$

(where $\left(a_{q+1} \ldots a_{q+p}\right)$ is the repeating part), then $\mathfrak{T}$ will have $q+p$ sets in the tile type partition denoted by $\mathfrak{T}_{j}, j \in\{1,2,3, \ldots, q+p\}$. Let $T_{1}$ be the line segment of $E_{u}$ with endpoints 0 and $e_{u}$. Let $T_{1} \in \mathfrak{T}_{1}$. Then $\phi T_{1}=\beta T_{1}$ has endpoints 0 and $\beta e_{u}$. Let $T_{1}+k e_{u} \in \mathfrak{T}_{1}$ for $k \in\left\{0,1,2,3, \ldots, a_{1}-1\right\}$. Let $\left[a_{1}, \beta\right] e_{u} \in \mathfrak{T}_{2}$. Let $T_{2}=\left[0, \beta-a_{1}\right] e_{u}$. Then $T_{2}+a_{1} \in \mathfrak{T}_{2}$. We have $\phi\left(T_{2}+a_{1}\right)=\beta T_{2}+\beta a_{1}=\left[a_{1} \beta, \beta^{2}\right] e_{u}$. Since $a_{2}$ is the greatest integer less than $\beta^{2}-\beta a_{1}$, we let

$$
T_{1}+\left(a_{1} \beta+k\right) e_{u} \in \mathfrak{T}_{1} \quad \text { for each } k \in\left\{0, \ldots, a_{2}-1\right\} .
$$

We let $\left[a_{1} \beta+a_{2}, \beta^{2}\right] e_{u} \in \mathfrak{T}_{3}$. Let $T_{3}=\left[0, \beta^{2}-a_{1} \beta-a_{2}\right] e_{u}$. We repeat this procedure for $j \in\{3,4, \ldots, q+p\}$. Since $\operatorname{carry}(\beta)$ is eventually repeating, we see that

$$
\beta^{q+p}-\beta^{q+p-1} a_{1}-\cdots-a_{q+p}=\beta^{q}-a_{1} \beta^{q-1}-\cdots-a_{q} .
$$


Note

$$
T_{q+p}=\left[0, \beta^{q+p-1}-\sum_{k=1}^{q+p-1} \beta^{q+p-1-k} a_{k}\right] e_{u}
$$

and

$$
\begin{aligned}
\beta\left(\beta^{q+p-1}-\sum_{k=1}^{q+p-1} \beta^{q+p-1-k} a_{k}\right) & =\beta^{q+p}-\sum_{k=1}^{q+p-1} \beta^{q+p-k} a_{k} \\
& =a_{q+p}+\beta^{q}-\sum_{k=1}^{q-1} \beta^{q-1-k} a_{k} .
\end{aligned}
$$

So if $T=[x, y] e_{u} \in \mathfrak{T}_{q+p}$, then $\phi T$ is subdivided into $a_{q+p}$ tiles in $\mathfrak{T}_{1}$ and 1 tile in $\mathfrak{T}_{q+1}$. That is, $T_{1}+(\phi x+k) e_{u} \in \mathfrak{T}_{1}$ for $k \in\left\{0, \ldots, a_{q+p}-1\right\}$ and $T_{q+1}+\left(\phi x+a_{q+p}\right) \in$ $\mathfrak{T}_{q+1}$.

This defines the subdivision rules for $\mathfrak{T}$ and the tile types. Since carry $(\beta)$ has an infinite number of nonzero terms, every tile in $\mathfrak{T}$ has an eventual image which contains a tile in $\mathfrak{T}_{1}$. Since $T_{1}$ is the only generator for $\mathfrak{T}$, we have that $\mathfrak{T}$ is mixing. Since there are a finite number of tile types and line segments may be arranged in only a finite number of ways in a bounded region of $X_{u}, \mathfrak{T}$ has a finite number of local patterns. Moreover, the endpoints of tiles in $\mathfrak{T}$ lie in $\mathbb{Z}[\beta] e_{u}$. Hence the endpoints are the projection of points in $\mathbb{Z}[\phi] e=\mathbb{Z}^{n}$. So $\mathfrak{T}$ is a $\pi_{u}\left(\mathbb{Z}^{n}\right)$-finite self similar tiling of $X_{u}$ with expansion map $\phi$. Let $\gamma$ be a generating tile map such that the left endpoint for each tile is its control point. Then $c(\mathfrak{T}) \subset \pi_{u}\left(\mathbb{Z}^{n}\right)$. The subdivision graph is given in Figure 4. Each edge $\alpha$ is labeled with $L(\alpha)$. Multiple edges from $v_{j}$ to $v_{0}$ are indicated with a thick arrow and the range of the labels is given. Since every infinite path in the graph corresponds to an element of the $\beta$-shift, we'll call this the subdivision graph for the $\beta$-shift.

We are interested to know when $\mathfrak{T}$ is a Markov tiling. We note that $\mathfrak{T}$ is certainly a Markov tiling if every element of $\mathbb{Z}^{n}$ which projects to $X_{u}$ has an eventual image which projects to a control point for $\mathfrak{T}$ or, equivalently, if every nonnegative element of $\mathbb{Z}[\beta]$ has a finite $\beta$-expansion.

Frougny and Solomyak have given sufficient conditions for the nonnegative elements of $\mathbb{Z}[\beta]$ to have finite $\beta$-expansions [7]. Let

$$
x^{n}-d_{1} x^{n-1}-\cdots-d_{n-1} x-d_{n}
$$

be the minimal polynomial for $\beta$. If

$$
d_{1} \geq d_{2} \geq \cdots \geq d_{n-1} \geq d_{n}=1,
$$

then every nonnegative element of $\mathbb{Z}[\beta]$ has a finite $\beta$-expansion. So $\mathfrak{T}$ is a Markov tiling. In this case we will show that the two sided extension of the $\beta$-shift is a symbolic representation for $\phi \bmod \mathbb{Z}^{n}$.

Let $\phi \in \mathrm{GL}(n, \mathbb{Z})$ be a hyperbolic automorphism of $\mathbb{R}^{n}$ with characteristic polynomial $\chi_{\phi}$. Suppose $\chi_{\phi}$ is the minimal polynomial for a Pisot number $\beta$. We apply the above construction to tile $X_{u}=[0, \infty) e_{u}$, for $e_{u}=\pi_{u} e$. The tiling $\mathfrak{T}$ is a $\pi_{u}\left(\mathbb{Z}^{n}\right)$-finite self similar tiling of $X_{u}$ with expansion map $\phi$. If $\mathbb{Z}[\phi] e=\mathbb{Z}^{n}$ and every non-negative element of $\mathbb{Z}[\beta]$ has a finite $\beta$-expansion, then $\mathfrak{T}$ is a Markov tiling. If every nonnegative element of $\mathbb{Z}[\beta]$ has a finite $\beta$-expansion but $\mathbb{Z}[\phi] e \neq \mathbb{Z}^{n}$, then $\mathfrak{T}$ induces a periodic tiling of $\mathbb{R}^{n} \bmod \mathbb{Z}[\phi] e$. 


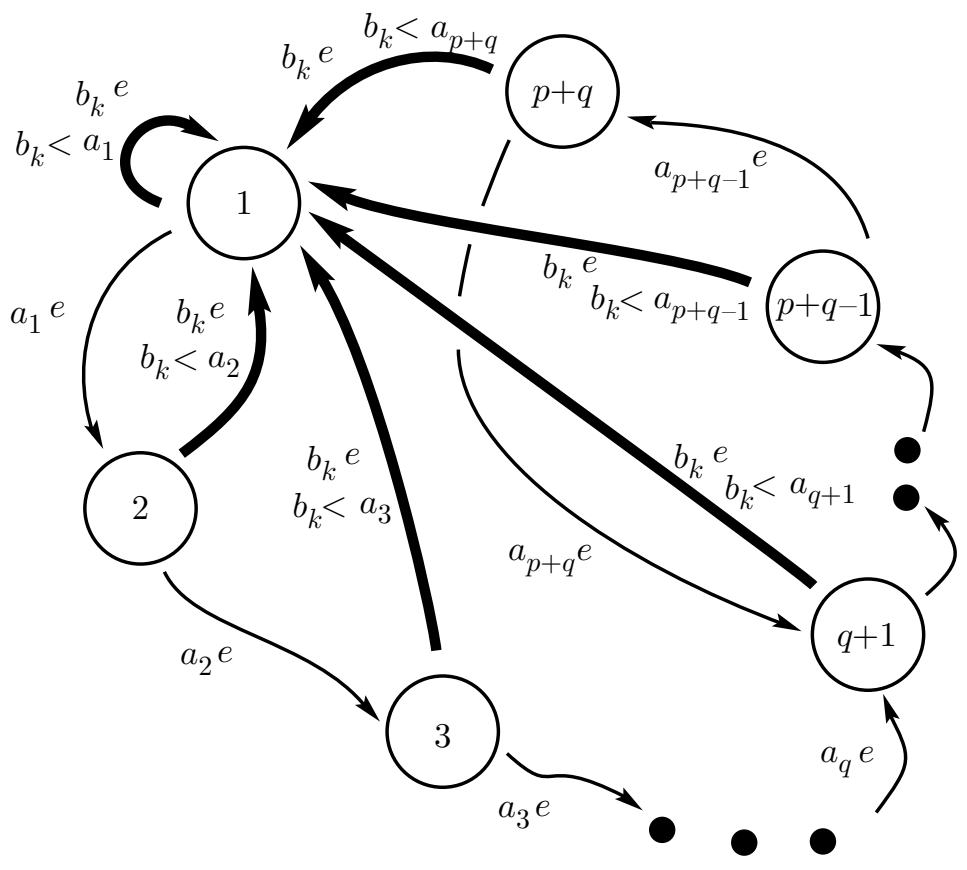

FIgURE 4. The subdivision graph for the $\beta$-shift.

Example 6. Let

$$
\phi=\left(\begin{array}{lll}
1 & 1 & 1 \\
1 & 0 & 0 \\
0 & 1 & 0
\end{array}\right) .
$$

Then $\phi$ has characteristic polynomial $x^{3}-x^{2}-x-1$. The eigenvalues for $\phi$ are $\lambda_{1}$, $\lambda_{2}, \lambda_{3}$ where $\lambda_{1}>1$ and $\lambda_{2}=\overline{\lambda_{3}}$ has modulus less than 1 . Note that $x^{3}-x^{2}-x-1$ satisfies the conditions of Frougny and Solomyak's theorem. Moreover $\mathbb{Z}[\phi] e=\mathbb{Z}^{n}$. So the corresponding self similar tiling is a Markov tiling. The carry sequence for $\lambda_{1}$ is (110). The subdivision graph is given in Figure 5. Each edge $\alpha$ is labeled with $L(\alpha)$.

The sets $\pi_{s}\left(\Omega_{1}\right), \pi_{s}\left(\Omega_{2}\right), \pi_{s}\left(\Omega_{3}\right)$ which arise out of this tiling coincide with the basic tiles found by Rauzy in [16]. Also note that $\pi_{s}\left(\Omega_{1}\right), \pi_{s}\left(\Omega_{2}\right), \pi_{s}\left(\Omega_{3}\right)$ are translates of the three basic tiles used in Bedford's construction (see [3]). A sketch of the sets $\Omega_{1}, \Omega_{2}, \Omega_{3}$ is in Figure 6 . The angles between the eigenvectors are distorted so that it is possible to see the rectangles. The bold lines indicate surfaces in front while the dotted lines indicate surfaces behind. Note that these really do look like crinkly tin cans.

Suppose now that $\mathbb{Z}[\phi] e$ is not equal to $\mathbb{Z}^{n}$ or that the nonnegative elements of $\mathbb{Z}[\beta]$ do not always have finite $\beta$-expansions. Then we must look for a self similar tiling different from the tiling given by the $\beta$-shift to construct a Markov partition for $\phi \bmod \mathbb{R}^{n}$. One such construction may be found in [15].

At this point we would like to say that every Markov partition consisting of rectangles which one finds in the literature may be constructed using self similar tilings. 


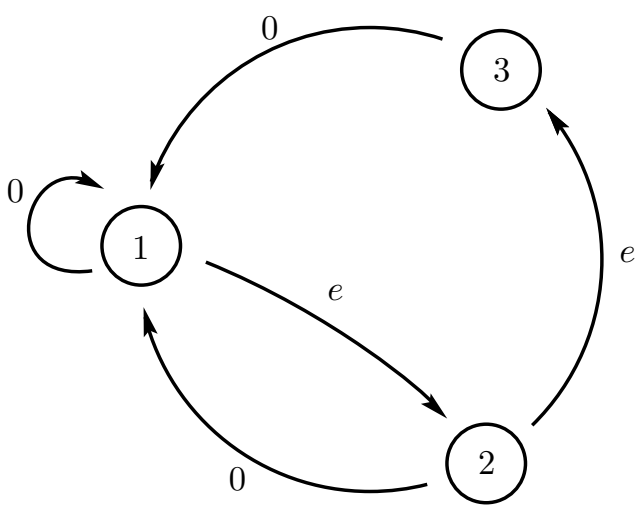

Figure 5. The subdivision graph for Example 5.3.

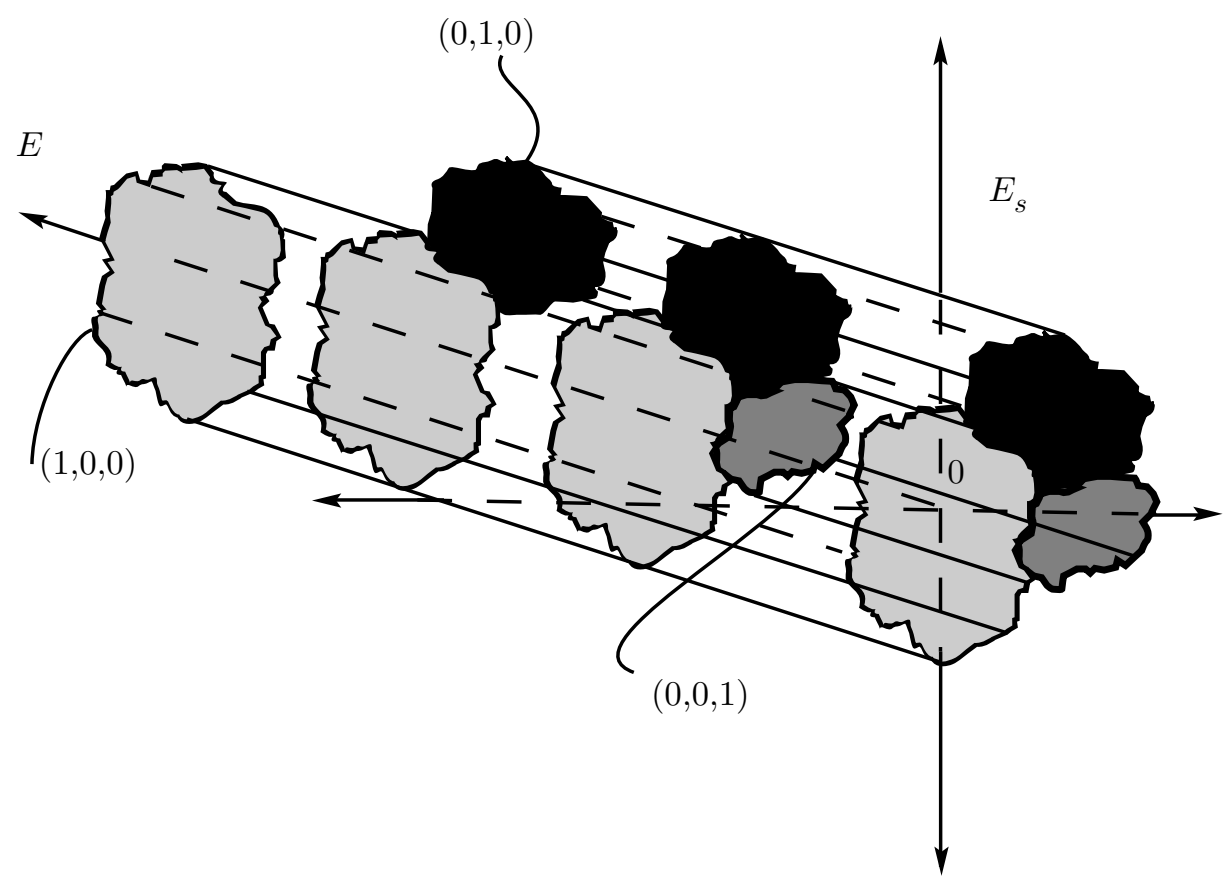

Figure 6. The periodic tiling in Example 5.3.

Unfortunately, things are never as simple as we would like and a more general construction using periodic self similar tilings is required. A complete treatment of the general case is found in [15].

We will end this section by proving Theorem 2.12 .

Proof of Theorem 2.12. Suppose that $X_{u}$ is a semigroup and for all $z \in\left(-X_{s} \oplus\right.$ $\left.X_{u}\right) \cap \mathbb{Z}^{n}$ there exists $N \geq 0$ such that $\phi^{N} z \in \zeta_{u}\left(c\left[P_{0}\right]\right)$. We will show that $\mathfrak{T}$ is a Markov tiling. 
The first step is to construct an open subset $U$ of $X_{s}$ which contains 0 in its closure such that $\phi U \subset U$ and if $z \in\left(-U \oplus X_{u}\right) \cap \mathbb{Z}^{n}$, then $z \in \zeta_{u} c\left[P_{0}\right]$. Suppose $z \in\left(-X_{s} \oplus X_{u}\right) \cap \mathbb{Z}^{n}$. By hypothesis $\pi_{u}(z)$ is the preimage of a control point. Hence there is a finite path $\left\{\eta^{k}\right\}_{k=-N}^{M}$ in $\Gamma$ with source in $\left\{v_{j}\right\}_{j \in J_{0}}$ such that $z=$ $\sum_{k=-N}^{M} \phi^{-k} L\left(\eta^{k}\right)$. By Lemma 2.10 there exists $N_{1}$ such that if $-\pi_{s} z \in \phi^{N_{1}} \pi_{s} \Omega$, then $M \leq 0$ and $z \in \zeta_{u} c(T)$. Let $T \in \mathfrak{T}$. By hypothesis there exists $N_{2}$ such that $\phi^{N_{2}} c(T) \in c\left[P_{0}\right]$. Since $\mathfrak{T}$ has a finite number of local patterns, we may choose $N_{2}$ so that for all $T \in \mathfrak{T}$ we have $\phi^{N_{2}} c(T) \in c\left[P_{0}\right]$. Hence if $-\pi_{s}(z) \in \phi^{N_{1}+N_{2}} \pi_{s} \Omega$, then $z \in \zeta_{u} c\left[P_{0}\right]$. Let $U=\phi^{N_{1}+N_{2}} \pi_{s} \Omega$.

The second step is to compensate for the possibility that $X_{u}$ may not be connected. Since $\left.\phi\right|_{E_{u}}$ is expansive, $X_{u}$ contains arbitrarily large connected components. If $T$ is a tile, then choose $N$ sufficiently large so that $T \subset \phi^{N} P_{0}$. Then for all $P \in\left[P_{0}\right], T+\phi^{N} c(P)$ is a tile of the same type as $T$. Moreover $T+\phi^{k+N} c(P)$ is a tile of the same type as $T$, for all $k \geq 0$. By the quasi-periodicity of $\mathfrak{T}$, each sufficiently large connected component of $X_{u}$ contains the pattern of $\phi^{N} P_{0}$ well within its interior. If $C$ is a connected component of $X_{u}$ which contains $\phi^{N} P$, then $\phi^{k} C$ is a connected component of $X_{u}$ which contains $\phi^{k+N} P$. Note that

$$
c\left(T+\phi^{k+N} c(P)\right)=c(T)+\phi^{k+N} c(P) .
$$

It follows that

$$
\left\|\rho_{s} c\left(T+\phi^{k+N} c(P)\right)-\rho_{s} c(T)\right\| \leq \varrho^{-k-N}\left\|\rho_{s} c(P)\right\| .
$$

Hence for every tile $T$ there exists a tile $T^{\prime}$ of the same type as $T$ and contained in an arbitrarily large connected component of $X_{u}$. Moreover, by fixing $P$ and letting $k$ get arbitrarily large, for any $\delta>0$, we may choose $T^{\prime}$ so that

$$
\left\|\rho_{s} c\left(T^{\prime}\right)-\rho_{s} c(T)\right\|<\delta .
$$

If $V$ is a bounded neighborhood of $\Omega$, then we choose $T^{\prime}$ so that

$$
\left(X_{u}-\zeta_{u} c\left(T^{\prime}\right)\right) \cap V=\left(E_{u}-\zeta_{u} c\left(T^{\prime}\right)\right) \cap V .
$$

Finally we suppose that $\mathfrak{T}$ is not a Markov tiling. The basic idea is to show that if for some distinct pair $j, k \in J$ and $z \in \mathbb{Z}^{n}-\{0\}$ we have

$$
\operatorname{Int}\left(\Omega_{j}\right) \cap \operatorname{Int}\left(\Omega_{k}\right)+z \neq \emptyset \text {, }
$$

then there exists $z^{\prime} \in \mathbb{Z}^{n}$ such that

$$
X_{u}-z^{\prime} \cap \operatorname{Int}\left(\pi_{s}\left(\Omega_{j}\right)\right) \neq \emptyset
$$

and $z^{\prime} \notin \zeta_{u} c\left(\mathfrak{T}_{j}\right)$. We construct a nonempty subset of $\left(-\operatorname{Int}\left(\pi_{s}\left(\Omega_{j}\right)\right) \oplus X_{u}\right) \cap \mathbb{Z}^{n}$ in which every point is not in $\zeta_{u} c\left(\mathfrak{T}_{j}\right)$. We then use the hypothesis of the theorem to show that such a set cannot exist.

So suppose for some distinct pair $j, k \in J$ and $z \in \mathbb{Z}^{n}-\{0\}$ we have

$$
\left(\operatorname{Int}\left(\Omega_{j}\right)+z\right) \cap \operatorname{Int}\left(\Omega_{k}\right) \neq \emptyset .
$$

Then for some $T \in \mathfrak{T}_{k}$ we have

$$
\left(T-\zeta_{u} c(T)\right) \cap\left(\operatorname{Int}\left(\Omega_{j}\right)+z\right) \neq \emptyset .
$$

By the above remarks we may choose $T$ so that

$$
\left(X_{u}-\zeta_{u} c(T)\right) \cap\left(\Omega_{j}+z\right)=\left(E_{u}-\zeta_{u} c(T)\right) \cap\left(\Omega_{j}+z\right) .
$$


So

$$
\left(X_{u}-\zeta_{u} c(T)-z\right) \cap \pi_{s}\left(\Omega_{j}\right)=\left\{\pi_{s}\left(-\zeta_{u} c(T)-z\right)\right\} .
$$

Since

$$
\left(X_{u}-\zeta_{u} c(T)-z\right) \cap \operatorname{Int}\left(\Omega_{j}\right) \neq \emptyset
$$

and $\Omega_{j}$ is a rectangle,

$$
\pi_{s}\left(-\zeta_{u} c(T)-z\right) \in \operatorname{Int}\left(\pi_{s}\left(\Omega_{j}\right)\right) \subset X_{s} .
$$

Moreover $z$ is within a bounded region of 0 . By choosing $T$ so that it lies well within a large connected ball in $X_{u}$ we have $c(T)+\pi_{u}(z) \in X_{u}$. Also $c(T)+\pi_{u}(z) \notin c\left(\mathfrak{T}_{j}\right)$. For if not, then $c(T)+\pi_{u}(z)=c\left(T^{\prime}\right)$ for some $T^{\prime} \in \mathfrak{T}_{j}$ and

$$
\left(X_{u}-\zeta_{u} c(T)-z\right) \cap \operatorname{Int}\left(\Omega_{j}\right)=\operatorname{Int}\left(T^{\prime}\right)-\zeta_{u} c\left(T^{\prime}\right) .
$$

Hence

$$
\left(T-\zeta_{u} c(T)-z\right) \cap\left(\operatorname{Int}\left(T^{\prime}\right)-\zeta_{u} c\left(T^{\prime}\right)\right) \neq \emptyset
$$

and $T \cap \operatorname{Int}\left(T^{\prime}\right) \neq \emptyset$. So $T=T^{\prime}$. But then $z=0$ and $j=k$.

It follows that if $\mathfrak{T}$ is not a Markov tiling, then there exist $j \in J$ and $z \in$ $\left(-X_{s} \oplus X_{u}\right) \cap \mathbb{Z}^{n}$ such that

$$
\left(X_{u}-z\right) \cap \operatorname{Int}\left(\pi_{s}\left(\Omega_{j}\right)\right) \neq \emptyset
$$

and $z \notin \zeta_{u} c\left(\mathfrak{T}_{j}\right)$.

Choose $M_{1}$ sufficiently large so that $\pi_{u}(z) \in \operatorname{Int}\left(\phi^{M_{1}} P_{0}\right)$. Then for all $P \in\left[P_{0}\right]$ we have $\zeta_{u} \phi^{M_{1}} c(P)+z \notin \zeta_{u} c\left(\mathfrak{T}_{j}\right)$. Hence for all $w \in\left(-\phi^{M_{1}} U \oplus X_{u}\right) \cap \mathbb{Z}^{n}$, we have $w+z \notin \zeta_{u} c\left(\mathfrak{T}_{j}\right)$ and

$$
\left(\left(-\phi^{M_{1}} U \oplus X_{u}\right)+z\right) \cap \zeta_{u} c\left(\mathfrak{T}_{j}\right)=\emptyset .
$$

Since $-\pi_{s}(z) \in \operatorname{Int}\left(\pi_{s} \Omega_{j}\right)$ and $0 \in \operatorname{Clos}\left(\phi^{M_{1}} U\right)$, there exists $T^{\prime \prime} \in \mathfrak{T}_{j}$ such that

$$
\rho_{s} c\left(T^{\prime \prime}\right) \in-\pi_{s}(z)+\phi^{M_{1}} U
$$

Choose $M_{2}$ sufficiently large so that

$$
T^{\prime \prime} \subset \phi^{M_{2}} P_{0},
$$

and so that, for all $P \in\left[P_{0}\right], \phi^{M_{2}} c\left(P_{0}\right)+c\left(T^{\prime \prime}\right) \in c\left(\mathfrak{T}_{j}\right)$ and

$$
\rho_{s}\left(\phi^{M_{2}} c(P)+c\left(T^{\prime \prime}\right)\right) \in\left(\left(\phi^{M_{1}} U-\pi_{s}(z)\right) \cap \rho_{s} c\left(\mathfrak{T}_{j}\right)\right) .
$$

Since $X_{u}$ is a semigroup, and $\pi_{u}(z) \in X_{u}$, we have

$$
X_{u}+\pi_{u}(z) \subset X_{u}
$$

Since $\mathfrak{T}$ is quasi-periodic, there exists $P \in\left[P_{0}\right]$ such that

$$
\phi^{M_{2}} P \subset X_{u}+\pi_{u}(z) .
$$

Hence $\phi^{M_{2}} c(P)+c\left(T^{\prime \prime}\right) \in X_{u}+\pi_{u}(z)$. So

$$
\zeta_{u} \phi^{M_{2}} c(P)+\zeta_{u} c\left(T^{\prime \prime}\right) \in\left(\left(-\phi^{M_{1}} U \oplus X_{u}\right)+z\right) \cap \zeta_{u} c\left(\mathfrak{T}_{j}\right)
$$

contradicting equation (3). It follows that $z \in \zeta_{u} c\left(\mathfrak{T}_{j}\right)$ and $\mathfrak{T}$ is a Markov tiling. 


\section{Symbolic REPRESENTATIONS}

We have seen that if $\mathfrak{T}$ is a Markov tiling with expansion map $\phi$, then we can wrap $\mathfrak{T}$ inside $\mathbb{R}^{n} \bmod \mathbb{Z}^{n}$ in such a way that the tiles of the same type form almost disjoint rectangles $\left\{\Omega_{j}\right\}_{j \in J}$. This is the first step to construct a Markov partition for $\phi \bmod \mathbb{Z}^{n}$. Once we have a Markov partition we will be able to represent the dynamical system $\left(\mathbb{R}^{n} \bmod \mathbb{Z}^{n}, \phi \bmod \mathbb{Z}^{n}\right)$ as a shift of finite type. In [1] Adler and Marcus outline the essential requirements for a symbolic system to represent an abstract dynamical system. In this final section we review these ideas. We show that if $\mathfrak{T}$ is a Markov tiling, then $\psi$ may be extended to a map from $\Sigma_{\Gamma}$ to $\mathbb{R}^{n} \bmod \mathbb{Z}^{n}$ in such a way that this extension meets the requirements for a symbolic representation. The following definitions come from [1, pp. 5-6]. We omit most proofs. Detailed proofs may be found in [15].

Definition. A dynamical system $(X, f)$ is said to be ergodically supported if there exists an ergodic $f$-invariant probability measure $\mu_{x}$ which is positive on open sets. Such measures are called ergodically supporting. A subset $N \subset X$ is called universally null if it has measure zero with respect to all ergodically supporting measures.

Definition. If $(X, f)$ and $(Y, h)$ are ergodically supported systems and $\pi$ is a map from $Y$ into $X$, then $\pi$ is called an almost homeomorphic factor map of $Y$ into $X$ if

(1) $\pi$ is onto,

(2) $\pi$ is boundedly finite to one,

(3) $\pi$ is continuous,

(4) $f \circ \pi=\pi \circ h$, and

(5) $\pi$ maps $Y-\pi^{-1}(N)$ one to one onto $X-N$ for some $f$ invariant universally null set $N$.

From now on we assume that $\mathfrak{T}$ is a Markov tiling of $X_{u}$. Define $\Pi: \mathbb{R}^{n} \rightarrow$ $\mathbb{R}^{n} \bmod \mathbb{Z}^{n}$ to be the quotient map which identifies points in $\mathbb{R}^{n}$ modulo the integer lattice. Since $\Omega$ is a fundamental region for a periodic tiling of $\mathbb{R}^{n} \bmod \mathbb{Z}^{n}$, a set $U \subset \Pi \Omega$ is open if and only if $\Pi^{-1} U \cap \Omega$ is open in the relative topology of $\Omega$. Let $\hat{\phi}: \Pi \Omega \rightarrow \Pi \Omega$ by $\hat{\phi}(\Pi x)=\Pi(\phi x)$. Let $\hat{\psi}: \Sigma_{\Gamma} \rightarrow \Pi \Omega$ by $\hat{\psi}(\eta)=\Pi \psi(\eta)$.

Theorem 3.1. The map $\hat{\psi}$ is an almost homeomorphic factor map from $\left(\Sigma_{\Gamma}, \sigma_{\Gamma}\right)$ to $(\Pi \Omega, \hat{\phi})$.

To prove this we note that the non-doubly transitive points in $(\Pi \Omega, \hat{\phi})$ form a universally null set. We construct a partition for $\Pi \Omega$ which will yield a generating set for a sigma-algebra on $\Pi \Omega$ which is metrically equivalent to the Borel sigma algebra. We show that every point whose orbit misses the boundary of this partition has a unique preimage in $\Sigma_{\Gamma}$. Finally we show that the doubly transitive points have orbits which miss the boundary of the partition. We call this partition a Markov partition.

Recall from the last section that $\psi$ is a continuous surjection from $\Sigma_{\Gamma}$ to $\Omega$. Hence $\Pi \circ \psi$ is a continous surjection from $\Sigma_{\Gamma}$ to $\Pi \Omega$.

Lemma 3.2. For all $k \in \mathbb{Z}$

$$
\hat{\phi}^{k} \circ \hat{\psi}=\hat{\psi} \circ \sigma_{\Gamma}^{k}
$$


Proof. This follows from Proposition 2.5.

Lemma 3.3. $\hat{\psi}$ is boundedly finite to one.

Proof. This follows from Proposition 2.7.

Proposition 3.4. Suppose $N, M \geq 0$ and $\left\{\eta^{k}\right\}_{k=-N}^{M}$ is a path in $\Gamma$ with source $v_{j_{0}}$ and target $v_{j_{1}}$. Then

$$
\begin{aligned}
& \psi\left(C_{-N}\left(\eta^{-N} \cdots \eta^{M}\right)\right) \\
& =\left(\phi^{N+1} \Omega_{j_{0}}-\sum_{k=-N}^{0} \phi^{-k} \zeta_{u} L\left(\eta^{k}\right)\right) \cap\left(\phi^{-M} \Omega_{j_{1}}+\sum_{k=1}^{M} \phi^{-k} \zeta_{u} L\left(\eta^{k}\right)\right) \\
& =\left(\pi_{s} \phi^{N+1} \Omega_{j_{0}}+\sum_{k=-N}^{0} \phi^{-k} \rho_{s} L\left(\eta^{k}\right)\right) \oplus\left(\pi_{u} \phi^{-M} \Omega_{j_{1}}+\sum_{k=1}^{M} \phi^{-k} L\left(\eta^{k}\right)\right),
\end{aligned}
$$

and $\psi\left(C_{-N}\left(\eta^{-N} \cdots \eta^{M}\right)\right)$ is the closure of its interior.

Proof. This follows from repeated applications of Proposition 2.5.

This proposition tells us all that we need to know about the $\psi$-images of the cylinder sets. By taking unions of sets of the form $C_{-N}\left(\eta^{-N} \cdots \eta^{M}\right)$ we may find the $\psi$-image of any cylinder set.

Definition. For each $\alpha \in \mathcal{E}$ let $R_{\alpha}=\psi C_{0}(\alpha)$. Let $\mathcal{R}=\left\{R_{\alpha}: \alpha \in \mathcal{E}\right\}$, and $\Pi \mathcal{R}=\left\{\Pi R_{\alpha}: \alpha \in \mathcal{E}\right\}$.

By Proposition 3.4, if $\alpha \in \mathcal{E}$ and $\Theta(\alpha)=\left(v_{j_{0}}, v_{j_{1}}, L(\alpha)\right)$, then

$$
\begin{aligned}
R_{\alpha} & =\left(\phi \Omega_{j_{0}}-\zeta_{u} L(\alpha)\right) \cap \Omega_{j_{1}} \\
& =\left(\pi_{s} \phi \Omega_{j_{0}}+\rho_{s} L(\alpha)\right) \oplus \pi_{u} \Omega_{j_{1}} .
\end{aligned}
$$

Proposition 3.5. (1) For each $\alpha \in \mathcal{E}$ the set $\Pi\left(\operatorname{Int}\left(R_{\alpha}\right)\right)$ is dense in $\operatorname{Int}\left(\Pi R_{\alpha}\right)$ and

$$
\begin{aligned}
\Pi R_{\alpha} & =\operatorname{Clos}\left(\Pi\left(\operatorname{Int}\left(R_{\alpha}\right)\right)\right) \\
& =C \operatorname{los}\left(\operatorname{Int}\left(\Pi R_{\alpha}\right)\right) .
\end{aligned}
$$

(2) $\Pi \mathcal{R}$ forms an almost disjoint cover of $\Pi \Omega$.

(3) For each $\alpha \in \mathcal{E}$ and $\eta \in \Sigma_{\Gamma}$ we have $\hat{\psi}(\eta) \in \operatorname{Int}\left(\Pi R_{\alpha}\right)$ only if $\eta^{0}=\alpha$.

Hence if $\hat{\psi}(\eta) \in \Pi\left(\operatorname{Int}\left(R_{\alpha}\right)\right)$, then $\eta^{0}=\alpha$.

It follows from (3) above that if $x \in \Omega$ such that, for all $k \in \mathbb{Z}, \Pi \phi^{k} x \in$ $\Pi\left(\operatorname{Int}\left(R_{\alpha}\right)\right)$ for some $\alpha \in \mathcal{E}$, then $\Pi x$ has a unique $\hat{\psi}$ preimage in $\Sigma_{\Gamma}$. We will show that the orbit of a doubly transitive point lies in

$$
\bigcup_{\alpha \in \mathcal{E}} \Pi\left(\operatorname{Int}\left(R_{\alpha}\right)\right) \text {. }
$$

The natural way to approach this is to consider the boundary of $R_{\alpha}$.

Definition. If $\alpha \in \mathcal{E}$ and $\Theta(\alpha)=\left(v_{j_{0}}, v_{j_{1}}, L(\alpha)\right)$, then the stable boundary of $R_{\alpha}$ is

$$
\partial^{s} R_{\alpha}=\left(\pi_{s} \phi \Omega_{j_{0}}+\rho_{s} L(\alpha)\right) \oplus \partial \pi_{u} \Omega_{j_{1}}
$$

and the unstable boundary of $R_{\alpha}$ is

$$
\partial^{u} R_{\alpha}=\left(\partial \pi_{s} \phi \Omega_{j_{0}}+\rho_{s} L(\alpha)\right) \oplus \pi_{u} \Omega_{j_{1}} .
$$




$$
\begin{gathered}
\text { If } x \in R_{\alpha}=\left(\pi_{s} \phi \Omega_{j_{0}}+\rho_{s} L(\alpha)\right) \oplus \pi_{u} \Omega_{j_{1}} \text { but } x \notin \partial^{s} R_{\alpha} \cup \partial^{u} R_{\alpha} \text {, then } \\
\pi_{s}(x) \in \operatorname{Int}\left(\pi_{s} \phi \Omega_{j_{0}}\right)+\rho_{s} L(\alpha)
\end{gathered}
$$

and

$$
\pi_{u}(x) \in \operatorname{Int}\left(\pi_{u} \Omega_{j_{1}}\right) .
$$

Hence $x \in \operatorname{Int}\left(R_{\alpha}\right)$. So $\partial R_{\alpha}=\partial^{s} R_{\alpha} \cup \partial^{u} R_{\alpha}$. Let $\partial^{s} \mathcal{R}=\bigcup_{\alpha \in \mathcal{E}} \partial^{s} R_{\alpha}$ and $\partial^{u} \mathcal{R}=\bigcup_{\alpha \in \mathcal{E}} \partial^{u} R_{\alpha}$. Then $\partial \mathcal{R}=\bigcup_{\alpha \in \mathcal{E}} \partial R_{\alpha}=\partial^{s} \mathcal{R} \cup \partial^{u} \mathcal{R}$. It follows from the definitions that:

Proposition 3.6. For each $\alpha \in \mathcal{E}$

(1) $\Pi \phi \partial^{s} R_{\alpha} \subset \Pi \partial^{s} \mathcal{R}$ and

(2) $\Pi \phi^{-1} \partial^{u} R_{\alpha} \subset \Pi \partial^{u} \mathcal{R}$.

Corollary 3.7. Let $\alpha \in \mathcal{E}$ and $\Theta(\alpha)=\left(v_{j_{0}}, v_{j_{1}}, L(\alpha)\right)$. Then

(1) $\phi R_{\alpha} \subset \bigcup_{\alpha^{\prime} \in \mathcal{E}_{j_{1}}}\left(R_{\alpha^{\prime}}+\zeta_{u} L\left(\alpha^{\prime}\right)\right)$,

(2) $\phi^{-1} R_{\alpha} \subset \bigcup_{\alpha^{\prime} \in \mathcal{E}^{j_{0}}} R_{\alpha^{\prime}}-\zeta_{u} \phi^{-1} L(\alpha)$,

(3) $\phi^{-1} \pi_{s} R_{\alpha}=\bigcup_{\alpha^{\prime} \in \mathcal{E}^{j} 0} \pi_{s} R_{\alpha^{\prime}}+\rho_{s} \phi^{-1} L(\alpha)$,

(4) $\phi \pi_{u} R_{\alpha}=\bigcup_{\alpha^{\prime} \in \mathcal{E}_{j_{1}}}\left(\pi_{u} R_{\alpha^{\prime}}+L\left(\alpha^{\prime}\right)\right)$

where these are almost disjoint unions.

Proof of Theorem 3.1.

Let $\mathfrak{N}=\Pi\left(\bigcup_{k \in \mathbb{Z}} \phi^{-k} \partial \mathcal{R}\right)=\bigcup_{k \in \mathbb{Z}} \hat{\phi}^{-k} \Pi \partial \mathcal{R}$. If $x \in \Omega$ and $\Pi x \in \Pi \Omega-\mathfrak{N}$, then for all $k \in \mathbb{Z}$

$$
\hat{\phi}^{k} \Pi x \notin \Pi \partial \mathcal{R} .
$$

Hence if $y_{k} \in \Omega$ such that $\Pi y_{k}=\hat{\phi}^{k} \Pi x$, then $y_{k} \notin \partial \mathcal{R}$. So for all $k \in \mathbb{Z}$ there exists $\eta^{k} \in \mathcal{E}$ such that

$$
\hat{\phi}^{k} \Pi x=\Pi y_{k} \in \Pi\left(\operatorname{Int}\left(R_{\eta^{k}}\right)\right) .
$$

By Proposition $3.5 \Pi x$ has a unique $\hat{\psi}$ preimage $\left\{\eta^{k}\right\}_{k \in \mathbb{Z}}$.

Suppose $\Pi x$ is doubly transitive. If for some $N \in \mathbb{Z}$ we have $\hat{\phi}^{N} \Pi x \in \Pi \partial \mathcal{R}$, then $\hat{\phi}^{N} \Pi x \in \Pi\left(\partial^{s} \mathcal{R} \cup \partial^{u} \mathcal{R}\right)$. Hence by Proposition $3.6 \Pi x$ is not doubly transitive. It follows that the doubly transitive points lie in $\Pi \Omega-\mathfrak{N}$ and therefore have unique preimages. Since almost every point is doubly transitive $\mathfrak{N}$ is a universally null set. Since $\hat{\psi}$ is a continuous surjection, with Lemmas 3.2 and 3.3 we have shown that $\hat{\psi}$ satisfies the conditions for an almost homeomorphic factor map.

\section{APPENDIX: INSPLitTing THE TILE TYPES}

Insplitting is a procedure which takes a graph $\Gamma$ and generates a new graph $\widehat{\Gamma}$ by splitting one set of edges sharing a common target into multiple sets and giving each of these sets a distinct target. In terms of the tiling, we will split a tile type into two or more sets and call each of these new sets a tile type.

If $T \in \mathfrak{T}$, then there is a unique $T^{\prime} \in \mathfrak{T}$ such that $\phi^{-1}(T) \subset T^{\prime}$. If $T \in \mathfrak{T}_{j}$ and $T^{\prime} \in \mathfrak{T}_{k}$, then there is a unique edge $\alpha(T) \in \mathcal{E}$ such that

$$
\alpha(T)=\Theta^{-1}\left(v_{k}, v_{j}, d(T)-\phi d\left(T^{\prime}\right)\right) .
$$

Note that $T \in \mathfrak{T}_{j}$ if and only if $\alpha(T) \in \mathcal{E}^{j}$. 
Let $j_{0} \in J$ and split $\mathcal{E}^{j_{0}}$ into two sets $\mathcal{E}^{j_{0}^{1}}$ and $\mathcal{E}^{j_{0}^{2}}$. Let $\widehat{J}=J-\left\{j_{0}\right\} \cup\left\{j_{0}^{1}, j_{0}^{2}\right\}$. Let $\widehat{\Gamma}$ be the graph with vertex set $\widehat{\mathcal{V}}$ indexed by $\widehat{J}$ and edge set $\widehat{\mathcal{E}}$ defined as follows. Suppose $\alpha$ is an edge in $\mathcal{E}, i \in\{1,2\}$, and $j, k \in J$.

- If $\alpha \in \mathcal{E}^{j_{0}^{i}}$ and $s(\alpha)=v_{j_{0}}$, then let $\widehat{\alpha_{1}}, \widehat{\alpha_{2}} \in \widehat{\mathcal{E}}$ such that

$$
\widehat{\alpha_{1}} \in \widehat{\mathcal{E}}_{j_{0}^{1}}^{i} \text { and } \widehat{\alpha_{2}} \in \widehat{\mathcal{E}}_{j_{0}^{2}}^{j_{0}^{i}} \text {. }
$$

- If $\alpha \in \mathcal{E}^{j_{0}^{i}}$ and $s(\alpha)=v_{k}$ for some $k \neq j_{0}$, then let

$$
\widehat{\alpha} \in \widehat{\mathcal{E}}_{k}^{j_{0}^{i}} .
$$

- If $\alpha \in \mathcal{E}_{j_{0}}^{k}$ for some $k \neq j_{0}$, then let $\widehat{\alpha_{1}}, \widehat{\alpha_{2}} \in \widehat{\mathcal{E}}$ such that

$$
\widehat{\alpha_{1}} \in \widehat{\mathcal{E}}_{j_{0}^{1}}^{k} \text { and } \widehat{\alpha_{2}} \in \widehat{\mathcal{E}}_{j_{0}^{2}}^{k} \text {. }
$$

- If $\alpha \in \mathcal{E}_{j}^{k}$ for some $j, k \neq j_{0}$, then let

$$
\widehat{\alpha} \in \widehat{\mathcal{E}}_{j}^{k} \text {. }
$$

This procedure is called a single insplitting of $\Gamma$ induced by the partition $\left\{\mathcal{E}^{j_{0}^{1}}, \mathcal{E}^{j_{0}^{2}}\right\}$. From the above construction we may compute a transition matrix for $\widehat{\Gamma}$, indexed by $\widehat{J} \times \widehat{J}$ given by

$$
\left\{\left|\widehat{\mathcal{E}}_{j_{1}}^{j_{2}}\right|\right\}_{j_{1}, j_{2} \in \hat{J}}
$$

Let $i \in\{1,2\}$.

- If $j_{1} \in\left\{j_{0}^{1}, j_{0}^{2}\right\}$ and $j_{2}=j_{0}^{i}$, then

$$
\left|\widehat{\mathcal{E}}_{j_{1}}^{j_{2}}\right|=\left|\mathcal{E}_{j_{0}} \cap \mathcal{E}^{j_{0}^{i}}\right| .
$$

- If $j_{1} \in J-\left\{j_{0}\right\}$ and $j_{2}=j_{0}^{i}$, then

$$
\left|\widehat{\mathcal{E}}_{j_{1}}^{j_{2}}\right|=\left|\mathcal{E}_{j_{1}} \cap \mathcal{E}^{j_{0}{ }^{i}}\right| .
$$

- If $j_{1} \in\left\{j_{0}^{1}, j_{0}^{2}\right\}$ and $j_{2} \in J-\left\{j_{0}\right\}$, then

$$
\left|\widehat{\mathcal{E}}_{j_{1}}^{j_{2}}\right|=\left|\mathcal{E}_{j_{0}}^{j_{2}}\right| .
$$

- If $j_{1}, j_{2} \in J-\left\{j_{0}\right\}$, then

$$
\left|\widehat{\mathcal{E}}_{j_{1}}^{j_{2}}\right|=\left|\mathcal{E}_{j_{1}}^{j_{2}}\right| .
$$

It follows that if $\left\{\widehat{\mathfrak{T}}_{j}\right\}_{j \in \hat{J}}$ is a tile type partition for $\mathfrak{T}$ which induces a transition matrix equal to the transition matrix for $\widehat{\Gamma}$, then $\widehat{\Gamma}$ is the subdivision graph corresponding to $\mathfrak{T}$ with the partition $\left\{\widehat{\mathfrak{T}}_{j}\right\}_{j \in \hat{J}}$.

Suppose $T^{1}$ and $T^{2}$ are distinct tiles in $\mathfrak{T}_{j_{0}}$ such that $\alpha\left(T^{1}\right) \neq \alpha\left(T^{2}\right)$. There exists a partition of $\mathcal{E}^{j_{0}}$ into two sets $\mathcal{E}^{j_{0}^{1}}$ and $\mathcal{E}^{j_{0}^{2}}$ such that $\alpha\left(T^{1}\right) \in \mathcal{E}^{j_{0}^{1}}$ and $\alpha\left(T^{2}\right) \in \mathcal{E}^{j_{0}^{2}}$. Let $\widehat{\Gamma}$ be the graph obtained for $\Gamma$ by applying the insplitting rule using this partition. Let $\widehat{\mathcal{E}}$ be the new edge set and $\widehat{J}=J-\left\{j_{0}\right\} \cup\left\{j_{0}^{1}, j_{0}^{2}\right\}$ index the new vertex set as in the rule.

For each $\alpha \in \mathcal{E}$ there exists a corresponding edge or pair of edges in $\widehat{\mathcal{E}}$. If $\alpha \in \mathcal{E}_{j}^{k}$ and $k \neq j_{0}$, then the corresponding edges in $\widehat{\mathcal{E}}$ have target $v_{k}$. If $\alpha \in \mathcal{E}^{j_{0}^{1}}$, then the 
corresponding edge or edges in $\widehat{\mathcal{E}}$ have target $v_{j_{0}^{1}}$. If $\alpha \in \mathcal{E}^{j_{0}^{2}}$, then the corresponding edges in $\widehat{\mathcal{E}}$ have target $v_{j_{0}^{2}}$. For each $j \in J-\left\{j_{0}\right\}$ let

$$
\hat{\mathfrak{T}}_{j}=\left\{T \in \mathfrak{T}: \alpha(T) \in \mathcal{E}^{j}\right\}=\mathfrak{T}_{j} .
$$

Let

$$
\hat{\mathfrak{T}}_{j_{0}^{1}}=\left\{T \in \mathfrak{T}: \alpha(T) \in \mathcal{E}^{j_{0}^{1}}\right\}
$$

and

$$
\hat{\mathfrak{T}}_{j_{0}^{2}}=\left\{T \in \mathfrak{T}: \alpha(T) \in \mathcal{E}^{j_{0}^{2}}\right\} .
$$

Then $\left\{\hat{\mathfrak{T}}_{j_{0}^{1}}, \hat{\mathfrak{T}}_{j_{0}^{2}}\right\}$ is just a partition of $\mathfrak{T}_{j_{0}}$ and $\mathfrak{T}$ is a $G$-finite tiling with respect to the partition $\left\{\hat{\mathfrak{T}}_{j}\right\}_{j \in \hat{J}}$.

Suppose that $T \in \hat{\mathfrak{T}}_{j} \subset \mathfrak{T}_{k}$. Let $\left\{T^{\widehat{\alpha}}\right\}_{\widehat{\alpha} \in \widehat{\mathcal{E}}_{k}}$ be the unique set of tiles such that

$$
\phi T=\bigcup_{\widehat{\alpha} \in \widehat{\mathcal{E}}_{k}} T^{\widehat{\alpha}}
$$

and $T^{\widehat{\alpha}} \in \hat{\mathfrak{T}}_{k_{\hat{\alpha}}}$ if and only if $t(\widehat{\alpha})=v_{k_{\hat{\alpha}}}$ in $\widehat{\Gamma}$.

For each $\widehat{\alpha} \in \widehat{\mathcal{E}}_{j}$ there is a corresponding $\alpha \in \mathcal{E}_{k}$. The target of $\widehat{\alpha}$ depends upon the target of $\alpha$. Hence the new tile type of $T^{\widehat{\alpha}}$ depends on the target of $\alpha$. So the new pattern of $\phi T$ is determined by the original tile type of $T$. In particular, the image of every tile in $\mathfrak{T}_{k}$ has the same pattern in the new tile type partition. It follows that if $P$ is a finite union of tiles in $\mathfrak{T}$, then the pattern of $P$ with respect to the partition $\left\{\hat{\mathfrak{T}}_{j}\right\}_{j \in \hat{J}}$ depends only on the pattern of $\phi^{-1} P$ with respect to the partition $\left\{\mathfrak{T}_{j}\right\}_{j \in J}$. Hence any subdividing properties that applied to $\mathfrak{T}$ using the original partition still apply using the new partition. Moreover, by the construction we see that the transition matrix corresponding to the new partition is the same as the transition matrix for $\widehat{\Gamma}$. Hence $\widehat{\Gamma}$ is the subdivision graph for $\mathfrak{T}$ with tile type partition $\left\{\widehat{\mathfrak{T}}_{j}\right\}_{j \in \hat{J}}$. Finally, we note that $T^{1}$ and $T^{2}$ have different tile types in this new tile type partition.

Proposition 1.9. Let $j \in J$, let $T_{1}$ and $T_{2}$ be distinct tiles in $\mathfrak{T}_{j}$. There exists a partition of $\mathfrak{T}_{j}$ into two sets $\hat{\mathfrak{T}}_{j_{1}}$ and $\hat{\mathfrak{T}}_{j_{2}}$ such that

$$
\left\{\mathfrak{T}_{k}: k \in J-\{j\}\right\} \cup\left\{\hat{\mathfrak{T}}_{j_{1}}, \hat{\mathfrak{T}}_{j_{2}}\right\}
$$

is a tile type partition for $\mathfrak{T}$ which preserves the subdividing properties of $\mathfrak{T}$. Moreover $T_{1} \in \hat{\mathfrak{T}}_{j_{1}}$ and $T_{2} \in \hat{\mathfrak{T}}_{j_{2}}$.

Proof. If $\alpha\left(T_{1}\right) \neq \alpha\left(T_{2}\right)$, then we are done by the preceding remarks. We suppose now that $\alpha\left(T_{1}\right)=\alpha\left(T_{2}\right)$.

There exist a unique sequence of tiles $\left\{T_{1}^{k}\right\}_{k=0}^{\infty}$ such that $\phi^{-k} T_{1} \subset T_{1}^{k}$ and a unique sequence of tiles $\left\{T_{2}^{k}\right\}_{k=0}^{\infty}$ such that $\phi^{-k} T_{2} \subset T_{2}^{k}$. Since $\phi$ is expansive, for some $M_{0} \geq 0$ we have $0 \in T_{1}^{N} \cap T_{2}^{N}$ for all $N \geq M_{0}$. We claim for some minimal $K_{0} \geq 1$ we have

$$
\alpha\left(T_{1}^{K_{0}}\right) \neq \alpha\left(T_{2}^{K_{0}}\right)
$$


Suppose for a moment this is true. Then the tile type of $T_{1}^{K_{0}}$ is the same as the tile type of $T_{2}^{K_{0}}$. Otherwise the source of $\alpha\left(T_{1}^{K_{0}-1}\right)$ would be different from the source of $\alpha\left(T_{2}^{K_{0}-1}\right)$ and

$$
\alpha\left(T_{1}^{K_{0}-1}\right) \neq \alpha\left(T_{2}^{K_{0}-1}\right)
$$

contradicting the minimality of $K_{0}$.

By the remarks preceding this proposition we may construct a single insplitting of $\Gamma$ to form $\widehat{\Gamma}^{1}$ and the resulting tile type partition for $\mathfrak{T}$ will give $T_{1}^{K_{0}}$ and $T_{2}^{K_{0}}$ different tile types.

The control points for $\mathfrak{T}$ are positional points for $\mathfrak{T}$ so we may define a label map for $\widehat{\Gamma}^{1}$ in terms of the controls points. We define an edge assignment $\alpha^{1}: \mathfrak{T} \rightarrow \widehat{\mathcal{E}}^{1}$ in terms of the label map for $\widehat{\Gamma}$. Since $T_{1}^{K_{0}}$ and $T_{2}^{K_{0}}$ have different tile types, there exists a minimal $K_{1}<K_{0}$ such that

$$
\alpha^{1}\left(T_{1}^{K_{1}}\right) \neq \alpha^{1}\left(T_{2}^{K_{1}}\right) .
$$

By applying induction we construct a finite sequence of insplittings to form $\widehat{\Gamma}^{N}$ with edge assignment $\alpha^{N}: \mathfrak{T} \rightarrow \mathcal{E}^{N}$. It follows that there is a decreasing sequence

$$
0<K_{N}<K_{N-1}<\cdots<K_{1}<K_{0}
$$

such that $\alpha^{N}\left(T_{1}^{K_{N}}\right) \neq \alpha^{N}\left(T_{2}^{K_{N}}\right)$. Hence for some $N \geq 1$ we have

$$
\alpha^{N}\left(T_{1}\right) \neq \alpha^{N}\left(T_{2}\right)
$$

So we are in a position to define a tile type partition for $\mathfrak{T}$ which will force $T_{1}$ and $T_{2}$ to have different tile types in their respective tilings.

Why does $K_{0}$ exist? Suppose for all $k \geq 1$ we have

$$
\alpha\left(T_{1}^{k}\right)=\alpha\left(T_{2}^{k}\right) .
$$

Then for all $N \geq 1$ we have $T_{1}^{N} \neq T_{2}^{N}$. Otherwise for some minimal $N_{0}>0$ we have $T_{1}^{N_{0}}=T_{2}^{N_{0}}$. Suppose this is the case and $T_{1}^{N_{0}}$ and $T_{2}^{N_{0}}$ are in $\mathfrak{T}_{j}$. Let $x_{1}=\phi^{-N_{0}} c\left(T_{1}\right)$ and $x_{2}=\phi^{-N_{0}} c\left(T_{2}\right)$. We apply the proof for Theorem 1.5 and find

$$
\begin{aligned}
x_{1} & =c\left(T_{1}^{N_{0}}\right)+\sum_{i=1}^{N_{0}} \phi^{-i} L\left(\alpha\left(T_{1}^{N_{0}-i}\right)\right) \\
& =c\left(T_{2}^{N_{0}}\right)+\sum_{i=1}^{N_{0}} \phi^{-i} L\left(\alpha\left(T_{2}^{N_{0}-i}\right)\right) \\
& =x_{2} .
\end{aligned}
$$

So $c\left(T_{1}\right)=c\left(T_{2}\right)$. By Corollary 1.4 we have $T_{1}=T_{2}$. But we assumed that $T_{1} \neq T_{2}$. So $\left\{T_{1}^{k}\right\}_{k=0}^{\infty}$ and $\left\{T_{2}^{k}\right\}_{k=0}^{\infty}$ are distinct sequences for which $T_{1}^{k} \neq T_{2}^{k}$ for any $k \geq 1$. Since $\alpha\left(T_{1}^{k-1}\right)=\alpha\left(T_{2}^{k-1}\right)$, we have

$$
c\left(T_{1}^{k-1}\right)-\phi c\left(T_{1}^{k}\right)=c\left(T_{2}^{k-1}\right)-\phi c\left(T_{2}^{k}\right) .
$$

So

$$
c\left(T_{1}^{k-1}\right)-c\left(T_{2}^{k-1}\right)=\phi\left(c\left(T_{1}^{k}\right)-c\left(T_{2}^{k}\right)\right)
$$

for all $k \geq 0$. Hence

$$
c\left(T_{1}^{k}\right)-c\left(T_{2}^{k}\right)=\phi^{-k}\left(c\left(T_{1}\right)-c\left(T_{2}\right)\right)
$$


for all $k \geq 0$. Since $T_{1} \neq T_{2}$, we have $c\left(T_{1}\right) \neq c\left(T_{2}\right)$. Since $\phi$ is a homeomorphism,

$$
\left\{\phi^{-k}\left(c\left(T_{1}\right)-c\left(T_{2}\right)\right)\right\}_{k=1}^{\infty}
$$

is a sequence of distinct points converging to 0 . But $\mathfrak{T}$ is locally finite and for all $N \geq$ $M_{0}$ we have $T_{1}^{N}$ and $T_{2}^{N}$ contain 0 . So $\left\{c\left(T_{1}^{k}\right)-c\left(T_{2}^{k}\right)\right\}_{k \geq M_{0}}$ contains a finite number of distinct differences and these are bounded away from zero. The contradiction implies for some $K_{0}, \alpha\left(T_{1}^{K_{0}}\right) \neq \alpha\left(T_{2}^{K_{0}}\right)$. This proves the proposition.

\section{REFERENCES}

1. Roy Adler and Brian Marcus, Topological entropy and equivalence of dynamical systems, Memoirs of the American Mathematical Society, (219), 1978. MR 83h:28027

2. Roy Adler and Benjamin Weiss, Similarities of automorphisms of the torus, Memoirs of the American Mathematical Society, (98), 1970. MR 41:1966

3. Timothy Bedford, Crinkly Curves, Markov Partitions and Dimension, Ph.D. Thesis, Warwick University, 1984.

4. Rufus Bowen, Markov partitions for Axiom A diffeomorphisms, American Journal of Mathematics, 92:725-747, August, 1970. MR 43:27400

5. Rufus Bowen, Markov partitions are not smooth, Proceedings of the American Mathematical Society, 71(1):130-132, August, 1978. MR 57:14055

6. Elise Cawley, Smooth markov partitions and toral automorphisms, Ergodic Theory Dynamical Systems, 11(4):633-651, 1991. MR 92k:58199

7. Christiane Frougny and Boris Solomyak, Finite beta-expansions, Ergodic Theory Dynamical Systems, 12:713-723, 1992. MR 94a:11123

8. William J. Gilbert, The fractal dimension of sets derived from complex bases, Canadian Mathematical Bulletin, 29:495-500, 1986. MR 88b:28014

9. Richard Kenyon, Inflationary tilings with similarity structure, Comment. Math. Helvetici, 69:169-198, 1994. MR 95e:52043

10. Richard W. Kenyon, Self-similar tilings, Technical report, Geometry Supercomputer Project, University of Minnesota, 1990. Research Report GCG 21.

11. D. E. Knuth, The Art of Computer Programming, Addison-Wesley, Reading, Mass., 1973. MR 56:4281

12. D. A. Lind, Dynamical properties of quasihyperbolic toral automorphisms, Ergodic Theory and Dynamical Systems, 2:49-68, 1982. MR 84g:28017

13. D. A. Lind and B. Marcus, An introduction to Symbolic Dynamics and Coding, Cambridge University Press, New York, 1995. MR 97a:58050

14. W. Parry, On the $\beta$-expansions of real numbers, Acta Mathematica, 11:401-417, 1960. MR 26:288

15. Brenda Praggastis, Markov Partitions for Hyperbolic Toral Automorphisms, Ph.D. thesis, University of Washington, 1994.

16. Gerard Rauzy, Nombres algebriques et substitutions, Bull. Soc. Math. France, 110:147-178, 1982. MR 84h:10074

17. William P. Thurston, Groups, tilings, and finite state automata, Lecture notes distributed in conjunction with the Colloquium Series, 1989. In AMS Colloquium lectures.

Department of Mathematics, University of Washington, Seattle, Washington 98195

E-mail address: praggast@sprynet.com 